\title{
Composição, concentração e classificação do transporte urbano de massa inteligente e sustentável em quatro cidades inteligentes
}

\author{
Composition, concentration and classification of smart and sustainable urban mass transport in
}

four smart cities

Composición, concentración y clasificación del transporte masivo urbano inteligente y sostenible en cuatro ciudades inteligentes

\section{Resumo}

As cidades inteligentes aperfeiçoam o seu sistema de transporte de massa através da sua oferta diversificada dos seus modos de transporte urbano para o transporte público. Existem opções de tecnologias inteligentes e sustentáveis que podem ser embarcadas e estarem disponíveis para utilização nesse sistema. A composição do sistema de transporte urbano de massa é um serviço público/privado e consorciado ofertado para uma demanda de cidadãos que visa a melhoria da mobilidade urbana. O transporte urbano de massa pode ser classificado partindo do levantamento de dados, tais como: os tipos de modos de transporte de massa existentes, as tecnologias inteligentes disponíveis e as fontes de energia aplicadas para a locomoção. Nesta pesquisa foi aplicado o método de estudo de caso múltiplo do sistema de transporte de massa, com o uso da técnica de triangulação de três bases de dados coletados em 2020. O objetivo deste estudo foi analisar e compreender a composição e a concentração dos sistemas de transporte urbano de massa de quatro cidades inteligentes com perfil metropolitano e classificar essas cidades inteligentes utilizando relatórios e painéis (dashboard) com o auxílio do software Power Business Intelligence (BI) da Microsoft como ferramenta de análise final. Considera-se que esses sistemas de transporte urbano de massa metropolitanos são construídos historicamente em estágios de evolução tecnológica e são implantados nas cidades inteligentes de acordo com as necessidades de mobilidade urbana das cidades, e podem ser classificados como mais ou menos diversos, mais ou menos tecnológicos e mais ou menos limpos ou poluentes.

Palavras-chave: Transporte de massa; Tecnologias; Cidades inteligentes; Sustentabilidade.

\begin{abstract}
Smart cities improve their mass transportation system through their diversified offering of urban modes of transportation for public transport. There are options of smart and sustainable technologies that can be embedded and available to be used in this system. The composition of the urban mass transportation system is a public/private and consortium service offered to a demand from citizens that aims to improve urban mobility. Urban mass transport can be classified based on data such as the types of existing mass transport modes, the smart technologies available, and the energy sources used for locomotion. In this research, the multiple case study method of the mass transportation system was applied, with the use of the triangulation technique of three databases collected in 2020 . The aim of this study was to analyze and understand the composition and concentration of urban mass transport systems in four smart cities with metropolitan profile and classify these smart cities using reports and dashboards with the aid of Power Business Intelligence (BI) software from Microsoft as the ultimate analysis tool. It is considered that these metropolitan urban mass transportation systems are historically built-in stages of technological evolution and are deployed in smart cities according to the urban mobility needs of the cities, and can be classified as more or less diverse, more or less technological, and more or less clean or polluting.
\end{abstract}

Keywords: Mass transport; Technologies; Smart cities; Sustainability. 


\begin{abstract}
Resumen
Las ciudades inteligentes mejoran su sistema de transporte masivo a través de su oferta diversificada de modos de transporte urbano para el transporte público. Hay opciones de tecnologías inteligentes y sostenibles que pueden integrarse y estar disponibles para su uso en este sistema. La composición del sistema de transporte urbano de masas es un servicio público/privado y consorciado que se ofrece a una demanda de los ciudadanos con el objetivo de mejorar la movilidad urbana. A el transporte masivo urbano puede clasificarse en función de la recogida de datos, tales como los tipos de modos de transporte masivo existentes, las tecnologías inteligentes disponibles y las fuentes de energía aplicadas para la locomoción. En esta investigación se aplicó el método de estudio de casos múltiples del sistema de transporte masivo, utilizando la técnica de triangulación de tres bases de datos recolectadas en 2020 . El objetivo de este estudio fue analizar y comprender la composición y concentración de los sistemas de transporte masivo urbano en cuatro ciudades inteligentes con perfil metropolitano y clasificar estas ciudades mediante informes y cuadros de mando con la ayuda del software Power Business Intelligence (BI) de Microsoft como herramienta de análisis final. Se considera que estos sistemas metropolitanos de transporte urbano masivo se construyen históricamente en etapas de evolución tecnológica y se despliegan en las ciudades inteligentes según las necesidades de movilidad urbana de las ciudades, pudiendo clasificarse como más o menos diversos, más o menos tecnológicos y más o menos limpios o contaminantes.
\end{abstract}

Palabras clave: Transporte masivo; Tecnologías; Ciudades inteligentes; Sostenibilidad.

\title{
1. Introdução
}

Uma cidade sustentável inteligente é aquela que dispõe da tecnologia da informação e de outras ferramentas a fim de aprimorar a qualidade de vida e dos serviços, protegendo as necessidades dos cidadãos e considerando a atuação econômica, social, ambiental e cultural (Akande, Cabral, Gomes \& Casteleyn, 2019). As cidades classificadas como inteligentes apresentam sistemas de transporte, soluções básicas de infraestrutura e soluções de serviços inteligentes orientados para as Tecnologias de Informação e Comunicação (TICs) (Kumar et al., 2019). Podem ser citadas como TICs no transporte público, por exemplo, os sistemas de informação de passageiros em tempo real, sistemas de notificação de chegada de ônibus, sistemas automáticos de localização de veículos e sistemas de prioridade de sinal de trânsito para a aplicação no transporte público (Erkollari \& Oberer, 2018).

Outro aspecto importante das cidades inteligentes é a sustentabilidade. As tecnologias sustentáveis no transporte público são a preocupação com as metas para a eliminação de veículos movidos por combustíveis fósseis e para a transição de fontes de energia alternativas e sustentáveis como a elétrica (International Energy Agency - IEA, 2020). Em diversos países, a transição para os ônibus elétricos no transporte público já é desenvolvida amplamente por metas e protocolos de sustentabilidade adotados por políticas públicas, e abordando ativamente os desafios do desenvolvimento sustentável em áreas urbanas (Burch \& Gilchrist, 2018). As megacidades estão comprometidas em colaborar com eficácia, compartilhando o conhecimento e promovendo ações mensuráveis e sustentáveis sobre as mudanças climáticas no transporte público (C40 CITIES, 2021).

O problema do presente estudo ressalta a importância de mapear e classificar as tecnologias disponíveis embarcadas ou não no sistema de transporte urbano como ônibus, Veículo Leve sobre Trilhos - VLT (tramway), metrôs e trens e outras possibilidades de modos de transportes coletivos necessários as cidades inteligentes. Essa classificação é importante para determinar quais cidades inteligentes utilizam as Tecnologias de Informação e Comunicação (TICs) no transporte de passageiros de massa. A maior concentração de tecnologias em uma cidade pode facilitar o fluxo da mobilidade urbana. Assim é importante saber como classificar as tecnologias para atender as demandas do transporte público de massa.

O objetivo deste artigo é classificar os sistemas de transporte urbano de massa como inteligentes e sustentáveis em cidades inteligentes, que visam o uso de TICs e a redução dos impactos ambientais em áreas urbanas por fontes energia renováveis ou não renováveis em combustíveis utilizados. A metodologia utilizada é de abordagem qualitativa, de método indutivo e tipo exploratório, utilizando o método de estudo de caso múltiplo de quatros cidades, sendo duas do continente europeu, Madri (Espanha) e Paris (França), e duas do continente sul-americano São Paulo (Brasil) e Buenos Aires (Argentina). 
A escolha se deve às cidades terem perfil urbano e metropolitano, com alta concentração de tecnologias e diversidade dos seus modos de transportes. O método de cruzamento de base de dados por triangulação foi aplicado para entender a composição desses sistemas de transporte. Este artigo é dividido nas seguintes seções: introdução e revisão da literatura, materiais e métodos, resultado e discussão do estudo de caso múltiplo dos sistemas de transporte urbano de massa inteligente e sustentável e revisão da literatura e finalizando com a seção de considerações finais.

\section{Materiais e Métodos}

A pesquisa traz o método de estudo de caso múltiplo com base na formação de evidências (Yin, 2014). Utilizou-se a técnica convergente de triangulação para a validação dos dados coletados (Denzin \& Lincoln, 2000), conforme Figura 1.

Figura 1. Processo Metodológico do Estudo de Caso Múltiplo com a Técnica de Triangulação de Dados.

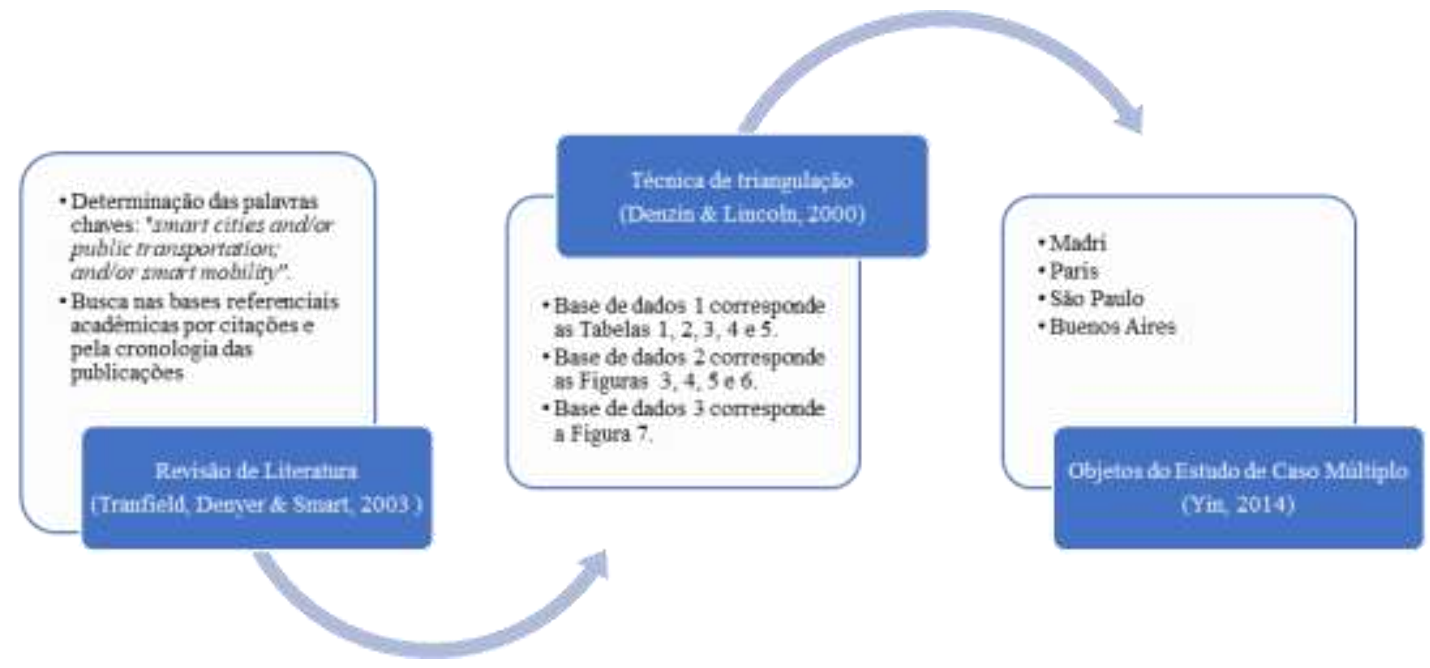

Fonte: Elaborado pelos autores (2021).

As seguintes etapas foram adotadas para a presente pesquisa:

(1) Realizou-se uma revisão de literatura utilizando as palavras-chaves em inglês: "smart cities and/or technologies and/or public transportation and/or mobility". As bases referenciadas consultadas foram: Science Direct, Emerald, IEEE XPLORE e DOAJ. Neste caso o método de revisão de literatura dá suporte ao método principal do estudo de caso múltiplo.

(2) As informações sobre o transporte público de massa para construção das três bases de dados foram coletadas dos websites das prefeituras, secretarias de economia, transporte e mobilidade, turismo e infraestrutura, e das empresas consorciadas em transporte público das quatro cidades pesquisadas, tendo sido os dados, portanto, coletados de formas diferentes para a triangulação da pesquisa (Flick, 1992). Os dados coletados na primeira base de dados trazem as informações econômicas, socioeconômicas, ranking das cidades inteligentes e a composição dos sistemas de transporte urbano de massa das quatro cidades. Na segunda base de dados compila-se e descreve-se a concentração dos modos de transporte público de massa, tecnologias e fontes de energia. Da terceira base de dados extraem-se as evidências para a proposta de "Classificação de classificação do sistema de transporte urbano de massa inteligente e sustentável em cidades inteligentes” (ver Figura 7).

(3) Cada cidade foi analisada e recebeu a palavra S, quando o dado coletado na investigação de triangulação estava presente; "N", quando o dado coletado na investigação de triangulação não estava presente, o que significa que o modo de transporte, a tecnologia ou fonte de energia não está disponível; e "NE" quando não foi encontrada informação sobre o dado coletado. Um asterisco “*” foi colocado quando constatada a ausência do modo de transporte de massa na investigação de triangulação; "NR" foi colocado para fonte de energia Não Renovável, e "R" para fonte de energia Renovável para o saldo de 
finalização da coleta de "Fontes de energia".

(4) Os dados dos modos de transporte e tecnologias inteligentes e sustentáveis foram transferidos para a criação dos painéis pelo software dashboard Power Business Intelligence (BI) da Microsoft, cujos resultados estão nas Figuras 3, 4, 5 e 6, que descrevem a concentração das tecnologias em cada modo de transporte de massa de cada cidade inteligente. Na Figura 7, são demonstrados os resultados para classificação das cidades inteligentes por volumes de tecnologias inteligentes e sustentáveis nos transportes de massa.

\section{Resultados e Discussão}

\subsection{Estudo de Caso Múltiplo do Transporte de Massa em Cidades Inteligentes}

Incialmente, nesta seção, são abordados os resultados das coletas dos dados para formação das bases de dados 1 e 2 de triangulação dos dados extraídos dos websites das prefeituras, das secretarias de transporte ou mobilidade urbana e das empresas consorciadas, nos quais geralmente são localizadas as informações econômicas, socioeconômicas e de transporte.

Tabela 1. Dados Econômicos, Socioeconômicos e Ranking das Cidades Inteligentes.

\begin{tabular}{|c|c|c|c|c|c|c|c|}
\hline $\mathbf{N}$ & Cidade & $\begin{array}{c}\text { População } \\
\text { Urbana }\end{array}$ & $\begin{array}{c}\text { População } \\
\text { Metropolitana }\end{array}$ & $\begin{array}{c}\text { Densidade } \\
\text { Populacional } \\
\text { Média } \\
\end{array}$ & IDH & $\begin{array}{c}\text { PIB da Cidade } \\
\text { (Bilhões U\$) }\end{array}$ & $\begin{array}{c}\text { Ranking } \\
\text { por Cidade } \\
\text { Inteligente }\end{array}$ \\
\hline 1 & Madri & 3.266 .126 & 6.791 .667 & $5.208,6$ & 0,992 & 308 & 71.42 \\
\hline 2 & Paris & 2.148 .271 & 12.628 .266 & 20.807 & 0,872 & 564 & 85.50 \\
\hline 3 & São Paulo & 12.252 .023 & 21.571 .281 & $13.024,56$ & 0,805 & 388 & 45.01 \\
\hline 4 & Buenos Aires & 2.891 .082 & 15.594 .428 & $14.307,68$ & 0,885 & 362 & 54.71 \\
\hline
\end{tabular}

Fonte: Portal web del Ayuntamiento de Madri (2020); Paris (2020); Prefeitura de São Paulo (2020); Buenos Aires Ciudad BA (2020); Country Economy (2020); IESE (2020); PricewaterhouseCoopers (2020).

A organização do fluxo da pesquisa aplicado à seção 2 de "Resultados e Discussões" é vista na Figura 2, a seguir:

Figura 2. Estrutura de Triangulação do Banco de Dados para os Estudos de Caso Múltiplos.

\begin{tabular}{|c|c|c|}
\hline $1^{\circ}$ Base de Dados & $2^{*}$ Base de Dados & $3^{*}$ Base de Dados \\
\hline $\begin{array}{c}\text { Tabela } 1 \\
\text { Dados econônicos, socioeconòmicos e } \\
\text { classificaçåo das cidades inteligentes } \\
\text { Tabeias } 2,3,4 \text { e } 5 \\
\text { Composiçầ đo sistema de transporte } \\
\text { urbano de massa em Maditi, Paris, São } \\
\text { Paulo e Buenos Aires }\end{array}$ & $\begin{array}{c}\text { Figuras 3, 4, } 5 \text { e } 6 \\
\text { Concentraçấo das tecnologias } \\
\text { inteligentes e fontes de energia para as } \\
\text { cidade de Madri, Paris, Săo Paulo e } \\
\text { Bunonos Aires } \\
\text { no estudo de caso múltiplo }\end{array}$ & $\begin{array}{l}\text { Figura } 7 \\
\text { Classificaçào do transporte de minssa } \\
\text { urbano inteligente e sustentavel para as } \\
\text { quatro cidades inteligentes analisadas }\end{array}$ \\
\hline & $\begin{array}{l}\text { Triangulacia dos dados para } \\
\text { estudo de caso múltiplo }\end{array}$ & \\
\hline
\end{tabular}

Fonte: Elaborado pelos autores (2021).

\subsubsection{Caracterização Estudo de Caso 1 (Cidade Inteligente de Madri)}

Madri é a maior economia da Espanha e a quarta a nível europeu, tendo como base o setor de serviços como os 
financeiros, o comércio e o turismo (Portal web del Ayuntamiento de Madrid, 2020). Geograficamente, está localizada na região central da Espanha, distante dos portos espanhóis. Estrategicamente, sua localização central auxilia a capilaridade das redes de transporte rodoviário, ferroviário e aeroviários de todo o país (Instituto Nacional de Estadística, 2020).

Sua região metropolitana com 6.791.667 habitantes (Tabela 1) abrange aos municípios de Alcorcón, Alcobendas, Coslada, Fuenlabrada, Getafe, Leganés, Móstoles, San Sebastián de los Reyes, San Fernando de Henares e Torrejón de Ardoz, que se tornam cidades-dormitório, compreendendo dez municípios limítrofes (Portal web del Ayuntamiento de Madrid, 2020).

O sistema de transporte público de transporte de massa de Madri possui sistemas de metrô, trem, ônibus urbano e interurbano, em que é explorada a concessão do transporte público/privado. O transporte público de Madri é gerenciado pelo "Consorcio Regional de Transporte Regulares de Madrid" (Consórcio Regional de Transporte de Madrid, 2020). O sistema de bilhetagem para o transporte público de massa é composto por vários tipos de bilhetes como (Movilidad y Transportes, 2020):

- Bilhete: utilizado para uma única viagem, sendo adquirido na mesma estação ou meio que pretende realizar. Em alguns serviços, como o trem de passageiros, podem ser adquiridos bilhetes de ida e volta.

- Bilhete Metrobús: é um bilhete válido tanto para a rede de metrô e rede de ônibus, permitindo a realização de um total de 10 viagens dentro da zona de transporte de Madri.

- Bilhete Abono transporte: é um bilhete de transporte válido para as redes de metrô, passageiros e ônibus urbanos ou interurbanos, permite a realização de um número ilimitado de viagens durante o seu período de validade, seja mensal ou anual, possuindo três modalidades (jovem, normal e terceira idade).

- Bilhete Abono turístico: bilhete válido entre um e sete dias, podendo incluir duas zonas: zona A, que é igual ao passe de transporte; e a zona T, que inclui todos eles. Suas taxas variam de acordo com a idade, definida entre normal (a partir dos 12 anos) e infantil (até 11 anos).

A Tabela 2 apresenta as tecnologias empregadas no transporte coletivo de transporte urbano de massa em Madri. 
Tabela 2. Composição do Sistema de Transporte Urbano de Massa de Madri.

\begin{tabular}{|c|c|c|c|c|c|c|c|c|}
\hline \multirow{2}{*}{$\frac{\text { Madri }}{\text { Tecnologia }}$} & \multicolumn{8}{|c|}{ Modos de Transporte Coletivo Público e Urbano de Massa } \\
\hline & $\begin{array}{c}\text { Ônibus a } \\
\text { Diesel }\end{array}$ & $\begin{array}{l}\text { Ônibus a } \\
\text { Gás }\end{array}$ & $\begin{array}{c}\text { Ônibus a } \\
\text { Bateria }\end{array}$ & Trólebus & VLT & Metrô & $\begin{array}{c}\text { Trem } \\
\text { Urbano }\end{array}$ & $\begin{array}{c}\text { Trem } \\
\text { Suburbano }\end{array}$ \\
\hline ITS & $\mathrm{S}$ & $\mathrm{S}$ & $\mathrm{S}$ & S & $\mathrm{S}$ & $\mathrm{S}$ & $\mathrm{S}$ & $\mathrm{S}$ \\
\hline Ar condicionado & $\mathrm{S}$ & $\mathrm{S}$ & $\mathrm{S}$ & $\mathrm{S}$ & $\mathrm{S}$ & $\mathrm{S}$ & $\mathrm{S}$ & S \\
\hline Internet 4G-usuário & $\mathrm{S}$ & $\mathrm{S}$ & S & $\mathrm{S}$ & $\mathrm{S}$ & $\mathrm{S}$ & $\mathrm{S}$ & S \\
\hline Internet 5G-usuário & $S$ & $\mathrm{~S}$ & $S$ & $\mathrm{~S}$ & $\mathrm{~S}$ & $\mathrm{~S}$ & $\mathrm{~S}$ & $S$ \\
\hline $\begin{array}{l}\text { Painéis de Informação de } \\
\text { horários e rotas }\end{array}$ & $S$ & $\mathrm{~S}$ & $\mathrm{~S}$ & $\mathrm{~S}$ & $\mathrm{~S}$ & $\mathrm{~S}$ & $\mathrm{~S}$ & $\mathrm{~S}$ \\
\hline Aplicativos-usuário & $S$ & $\mathrm{~S}$ & $\mathrm{~S}$ & $\mathrm{~S}$ & $\mathrm{~S}$ & $\mathrm{~S}$ & $\mathrm{~S}$ & $S$ \\
\hline $\begin{array}{l}\text { Registrador Mecânico de } \\
\text { passageiros }\end{array}$ & $\mathrm{N}$ & $\mathrm{N}$ & $\mathrm{N}$ & $\mathrm{N}$ & $\mathrm{N}$ & $\mathrm{N}$ & $\mathrm{N}$ & $\mathrm{N}$ \\
\hline $\begin{array}{l}\text { Registrador Eletrônico de } \\
\text { embarques e desembarques }\end{array}$ & $\mathrm{S}$ & $\mathrm{S}$ & $\mathrm{S}$ & $S$ & $\mathrm{~S}$ & $\mathrm{~S}$ & $\mathrm{~S}$ & $\mathrm{~S}$ \\
\hline $\begin{array}{l}\text { Emissor de comprovante } \\
\text { de pagamento }\end{array}$ & $\mathrm{S}$ & $\mathrm{S}$ & $\mathrm{S}$ & $\mathrm{S}$ & $\mathrm{S}$ & $\mathrm{S}$ & $\mathrm{S}$ & $\mathrm{S}$ \\
\hline Bilhete magnético & $S$ & $S$ & $\mathrm{~S}$ & $\mathrm{~S}$ & $\mathrm{~S}$ & $\mathrm{~S}$ & $\mathrm{~S}$ & $S$ \\
\hline Cartão inteligente & $S$ & $\mathrm{~S}$ & $\mathrm{~S}$ & $\mathrm{~S}$ & $\mathrm{~S}$ & $\mathrm{~S}$ & $\mathrm{~S}$ & $S$ \\
\hline Rádio Comunicador & $S$ & $\mathrm{~S}$ & $\mathrm{~S}$ & $\mathrm{~S}$ & $\mathrm{~S}$ & $\mathrm{~S}$ & $S$ & $S$ \\
\hline Identificador de Coletivos & $\mathrm{S}$ & $\mathrm{S}$ & $\mathrm{S}$ & $\mathrm{S}$ & $\mathrm{S}$ & $\mathrm{S}$ & $\mathrm{S}$ & $\mathrm{S}$ \\
\hline Registrador de passagem & $\mathrm{S}$ & $\mathrm{S}$ & $\mathrm{S}$ & $\mathrm{S}$ & $\mathrm{S}$ & $\mathrm{S}$ & $\mathrm{S}$ & $\mathrm{S}$ \\
\hline $\begin{array}{l}\text { Registrador de coletivos } \\
\text { por satélite }\end{array}$ & $\mathrm{S}$ & $\mathrm{S}$ & $S$ & $\mathrm{~S}$ & $\mathrm{~S}$ & $\mathrm{~S}$ & $\mathrm{~S}$ & $S$ \\
\hline $\begin{array}{l}\text { Registrador do } \\
\text { comportamento do } \\
\text { condutor e do veículo }\end{array}$ & $S$ & $\mathrm{~S}$ & $\mathrm{~S}$ & $\mathrm{~S}$ & $\mathrm{~S}$ & $\mathrm{~S}$ & $\mathrm{~S}$ & $S$ \\
\hline Registrador de Viagens & $\mathrm{S}$ & $\mathrm{S}$ & $S$ & $\mathrm{~S}$ & $\mathrm{~S}$ & $S$ & $S$ & $S$ \\
\hline Sinalização Braile & $\mathrm{S}$ & $\mathrm{S}$ & $\mathrm{S}$ & $\mathrm{S}$ & $\mathrm{S}$ & $\mathrm{S}$ & $\mathrm{S}$ & $\mathrm{S}$ \\
\hline Biometria Facial & $\mathrm{S}$ & $\mathrm{S}$ & $\mathrm{S}$ & $\mathrm{S}$ & $\mathrm{S}$ & $\mathrm{S}$ & $S$ & $\mathrm{~S}$ \\
\hline Comunicador-usuário & $\mathrm{S}$ & $\mathrm{S}$ & $\mathrm{S}$ & $\mathrm{S}$ & $\mathrm{S}$ & $\mathrm{S}$ & $\mathrm{S}$ & $\mathrm{S}$ \\
\hline Acionador de semáforo & $\mathrm{N}$ & $\mathrm{N}$ & $\mathrm{N}$ & $\mathrm{N}$ & $\mathrm{N}$ & $\mathrm{N}$ & $\mathrm{N}$ & $\mathrm{N}$ \\
\hline Vigiador de vias e interior & $\mathrm{S}$ & $\mathrm{S}$ & $\mathrm{S}$ & $\mathrm{S}$ & $\mathrm{S}$ & $\mathrm{S}$ & $\mathrm{S}$ & $\mathrm{S}$ \\
\hline $\begin{array}{l}\text { Global Positioning System } \\
\text { (GPS) }\end{array}$ & $S$ & $S$ & $\mathrm{~S}$ & $\mathrm{~S}$ & $\mathrm{~S}$ & $\mathrm{~S}$ & $\mathrm{~S}$ & $\mathrm{~S}$ \\
\hline Big Data & $\mathrm{S}$ & $\mathrm{S}$ & $\mathrm{S}$ & $\mathrm{S}$ & $\mathrm{S}$ & $\mathrm{S}$ & $S$ & $\mathrm{~S}$ \\
\hline Data Mining & $\mathrm{S}$ & $\mathrm{S}$ & $\mathrm{S}$ & $\mathrm{S}$ & $\mathrm{S}$ & $\mathrm{S}$ & $\mathrm{S}$ & $\mathrm{S}$ \\
\hline Cloud Computing & $\mathrm{S}$ & $\mathrm{S}$ & $\mathrm{S}$ & $\mathrm{S}$ & $\mathrm{S}$ & $\mathrm{S}$ & $\mathrm{S}$ & $\mathrm{S}$ \\
\hline Internet das Coisas & $\mathrm{S}$ & $\mathrm{S}$ & $\mathrm{S}$ & $\mathrm{S}$ & $\mathrm{S}$ & $\mathrm{S}$ & $\mathrm{S}$ & $\mathrm{S}$ \\
\hline Internet dos Serviços & $\mathrm{S}$ & $\mathrm{S}$ & $\mathrm{S}$ & $\mathrm{S}$ & $\mathrm{S}$ & $\mathrm{S}$ & $\mathrm{S}$ & $\mathrm{S}$ \\
\hline Crowdsourcing & $\mathrm{S}$ & $\mathrm{S}$ & $\mathrm{S}$ & $\mathrm{S}$ & $\mathrm{S}$ & $\mathrm{S}$ & $\mathrm{S}$ & $\mathrm{S}$ \\
\hline Blockchain & $\mathrm{NE}$ & $\mathrm{NE}$ & $\mathrm{NE}$ & $\mathrm{NE}$ & $\mathrm{NE}$ & $\mathrm{NE}$ & $\mathrm{NE}$ & $\mathrm{NE}$ \\
\hline Segurança Cibernética & $\mathrm{S}$ & $\mathrm{S}$ & $\mathrm{S}$ & $\mathrm{S}$ & $\mathrm{S}$ & $\mathrm{S}$ & $\mathrm{S}$ & $\mathrm{S}$ \\
\hline Sistemas integrados & $\mathrm{S}$ & $\mathrm{S}$ & $S$ & $\mathrm{~S}$ & $\mathrm{~S}$ & $\mathrm{~S}$ & $\mathrm{~S}$ & $S$ \\
\hline Sanitização & $\mathrm{S}$ & $\mathrm{S}$ & $\mathrm{S}$ & $\mathrm{S}$ & $\mathrm{S}$ & $\mathrm{S}$ & $\mathrm{S}$ & $\mathrm{S}$ \\
\hline Total de Tecnologias & 30 & 22 & 22 & 22 & 22 & 22 & 22 & 22 \\
\hline Tipos de Energia & Diesel & Gas & Bateria & Elétrica & Elétrica & Elétrica & Elétrica & Elétrica \\
\hline Fontes de Energia & NR & $\mathrm{R}$ & $\mathrm{R}$ & $\mathrm{R}$ & $\mathrm{R}$ & $\mathrm{R}$ & $\mathrm{R}$ & $\mathrm{R}$ \\
\hline
\end{tabular}

Fonte: Elaborado pelos autores (2021).

Na Figura 3 é apresentada a concentração das tecnologias e fontes de energia empregadas no transporte coletivo de transporte urbano de massa de Madri. 
Figura 3. Concentração de Tecnologias Inteligentes e Fontes de Energia para o Transporte de Massa da Cidade De Madri.

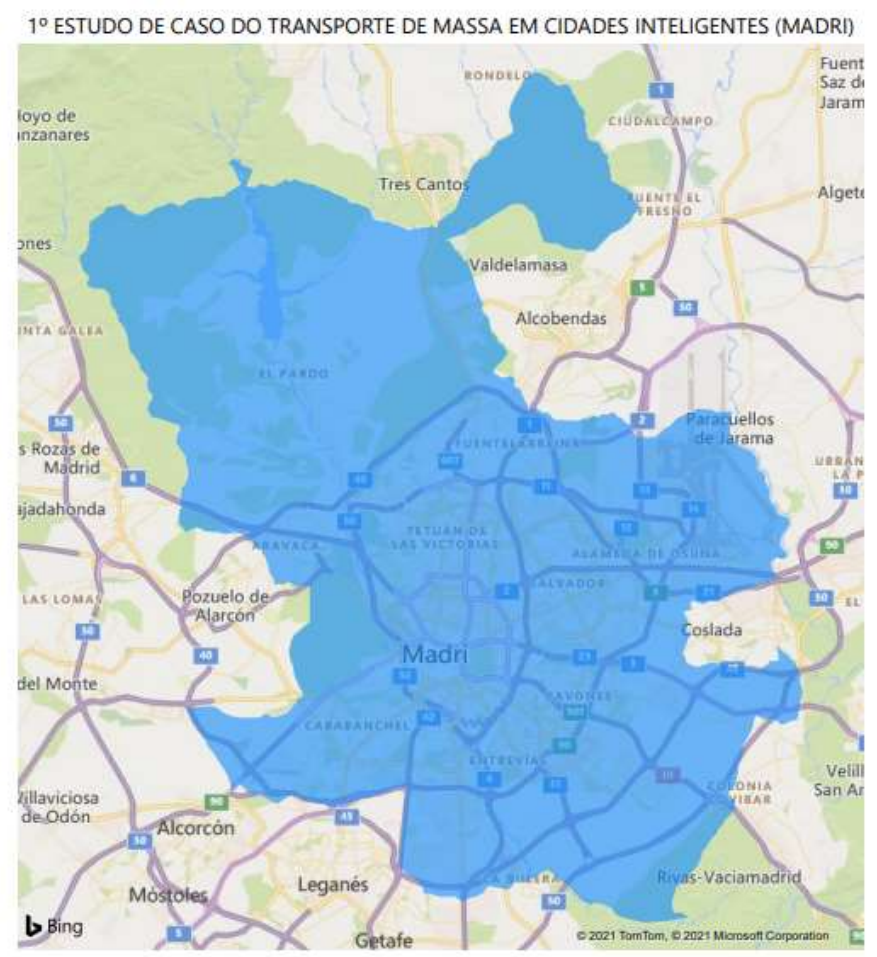
CONCENTRAÇĀO DAS TECNOLOGIAS INTELIGENTES NO TRANSPORTE DE MASSA

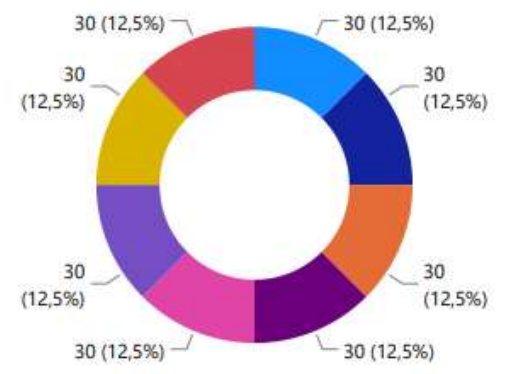

MODOS DE TRANSPORTE - METRÓ

- ÓNIBUs à Bateria - ÓNIBUS À dIESEL

- ÓNIBUS À GÁs

- TREM METROPOUTANO

- TREM URBANO

- TRÓLEBUS

- VLT

\section{CONCENTRAÇĀO DAS FONTES DE ENERGIA NO TRANSPORTE DE MASSA}

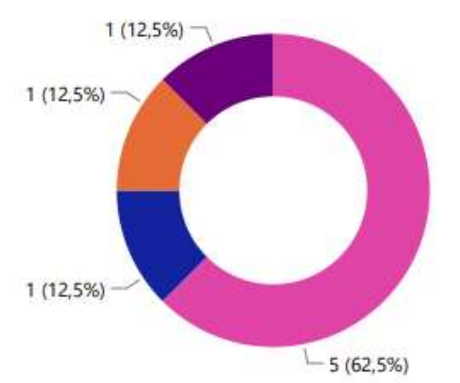

FONTES DE ENERGIA
ELETRIFICADA
- BATERIA
- DIESEL
- GÁS

Fonte: Elaborado pelos autores (2021).

Pode-se observar na Figura 3 que a concentração das tecnologias inteligentes no transporte de massa está equilibrada em quantidade e percentuais nos modos de transporte, de forma que se torna uma estratégia para melhorar a comunicação das tecnologias na mobilidade urbana da cidade inteligente de Madri. Sobre a concentração das fontes de energia no transporte de massa, o uso maior ocorre em energias renováveis buscando a sustentabilidade através da redução da poluição atmosférica e sonora nas cidades inteligentes. Madri tem como meta a redução gradual ou a proibição de todos os tipos de veículos a diesel a partir de 2025 e tem como "poderes fortes" da prefeitura a definição e aplicação das políticas no transporte público e como “poderes parciais” o controle orçamentário e de receita e visão definida no transporte público (C40 CITIES, 2021).

A vulnerabilidade do seu sistema de transporte de massa está na adesão da fonte de energia elétrica para ônibus. Suas tecnologias inteligentes e fontes de energia de transporte de massa são similares às da cidade inteligente de Paris.

\subsubsection{Caracterização Estudo de Caso 2 (Cidade Inteligente de Paris)}

Paris é a maior economia da França tendo como base o setor de lazer e turismo, de serviços (educação) e o comércio. Geograficamente, está localizada na região norte da França, distante dos portos franceses. Estrategicamente sua localização central auxilia a capilaridade das redes de transporte rodoviário, ferroviário e aeroviários de todo o país, e também, do Reino Unido e dos Países Baixos (Holanda, Bélgica e Luxemburgo) (Paris, 2020).

Sua região metropolitana, com 12.628.266 habitantes (Tabela 1) compreende os estabelecimentos públicos territoriais de Vallée Sud Grand Paris (VSGP), Grand Paris Seine Ouest (GPSO), Paris Ouest La Défense (POLD), Boucle Nord de Seine (BNS), Plaine Commune (PC), Paris Terres d'Envol (PTE), Est Ensemble (EE), Grand Paris - Grand Est (GPGE), Paris-Est-Marne et Bois (PEMB), Grand Paris Sud Est Avenir (GPSEA0 e Grand-Orly Seine Bièvre (GOSB), que se tornam cidades-dormitório, tendo dez municípios limítrofes (Paris, 2020). 
O sistema de transporte público de massa de Paris possui o metrô, o VLT, o trem, o ônibus urbano e interurbano. Esse sistema é gerenciado pelo conjunto das seguintes empresas consorciadas: Métro de Paris, para o sistema de metrô; Réseau Express Régional d'Île-de-France (RER), para o sistema de trem urbano; Tramway d'Île-de-France, para o sistema de VLTs; Transilien, para o sistema de trem metropolitano, Réseau de bus (RATP) e Autobus d'Île-de-France, para o sistema de linhas de ônibus de Paris para os subúrbios: Ille-de-France Mobilités que organiza os serviços de transporte público regular de passageiros, à procura e escolar, serviços de compartilhamento de bicicletas self-service e a Grand Paris Express que é uma de rede de metrô composta por quatro linhas automáticas em torno de Paris e duas extensões de linhas (Plan Paris $360^{\circ}$, 2020).

O sistema de bilhetagem para o transporte público de massa é composto por vários tipos de bilhetes como:

- t+ Ticket: bilhetagem básica, em que o valor é acrescido conforme a zona de desembarque pela empresa RATP, geralmente a cartela mais utilizada é de 10 passes.

- Travel Pass: é um bilhete para o turista pode ser adquirido com validade de 1 a 5 dias e disponibiliza viagens ilimitadas dentro das zonas compradas de 1 a 3 (região central) e 1 a 5 (subúrbios, aeroporto e Disney).

- Navigo Découverte: é um bilhete que oferece viagens ilimitadas e intermodais pelo sistema de transporte público.

A Tabela 3 apresenta as tecnologias empregadas no transporte coletivo de transporte urbano de massa em Paris. 
Tabela 3. Composição do Sistema de Transporte Urbano de Massa de Paris.

\begin{tabular}{|c|c|c|c|c|c|c|c|c|}
\hline \multirow{2}{*}{$\frac{\text { Paris }}{\text { Tecnologia }}$} & \multicolumn{8}{|c|}{ Modos de Transporte Coletivo Público e Urbano de Massa } \\
\hline & $\begin{array}{l}\text { Ônibus a } \\
\text { Diesel }\end{array}$ & $\begin{array}{l}\text { Ônibus } \\
\text { a Gás }\end{array}$ & $\begin{array}{c}\text { Ônibus a } \\
\text { Bateria }\end{array}$ & Trólebus & VLT & Metrô & $\begin{array}{c}\text { Trem } \\
\text { Urbano }\end{array}$ & $\begin{array}{c}\text { Trem } \\
\text { Suburbano }\end{array}$ \\
\hline ITS & $\mathrm{S}$ & $\mathrm{S}$ & $\mathrm{S}$ & $\mathrm{S}$ & $\mathrm{S}$ & $\mathrm{S}$ & $\mathrm{S}$ & $\mathrm{S}$ \\
\hline Ar condicionado & $\mathrm{S}$ & $\mathrm{S}$ & S & $\mathrm{S}$ & $\mathrm{S}$ & $\mathrm{S}$ & $\mathrm{S}$ & $\mathrm{S}$ \\
\hline Internet 4G-usuário & $\mathrm{S}$ & $\mathrm{S}$ & S & $\mathrm{S}$ & $\mathrm{S}$ & $\mathrm{S}$ & $\mathrm{S}$ & $\mathrm{S}$ \\
\hline Internet 5G-usuário & $\mathrm{S}$ & $S$ & $S$ & $\mathrm{~S}$ & $S$ & $S$ & $S$ & $\mathrm{~S}$ \\
\hline $\begin{array}{l}\text { Painéis de Informação de } \\
\text { horários e rotas }\end{array}$ & $\mathrm{S}$ & $S$ & S & $\mathrm{S}$ & $S$ & $\mathrm{~S}$ & $S$ & $\mathrm{~S}$ \\
\hline Aplicativos-usuário & $\mathrm{S}$ & $\mathrm{S}$ & $S$ & $\mathrm{~S}$ & $\mathrm{~S}$ & $\mathrm{~S}$ & $S$ & $\mathrm{~S}$ \\
\hline $\begin{array}{l}\text { Registrador Mecânico de } \\
\text { passageiros }\end{array}$ & $\mathrm{N}$ & $\mathrm{N}$ & $\mathrm{N}$ & $\mathrm{N}$ & $\mathrm{N}$ & $\mathrm{N}$ & $\mathrm{N}$ & $\mathrm{N}$ \\
\hline $\begin{array}{l}\text { Registrador Eletrônico de } \\
\text { embarques e desembarques }\end{array}$ & $\mathrm{S}$ & S & S & $\mathrm{S}$ & S & S & S & $\mathrm{S}$ \\
\hline $\begin{array}{l}\text { Emissor de comprovante } \\
\text { de pagamento }\end{array}$ & $\mathrm{S}$ & S & S & $\mathrm{S}$ & $S$ & S & S & $\mathrm{S}$ \\
\hline Bilhete magnético & $\mathrm{S}$ & $S$ & $S$ & $\mathrm{~S}$ & $S$ & $S$ & $S$ & $\mathrm{~S}$ \\
\hline Cartão inteligente & $\mathrm{S}$ & $\mathrm{S}$ & $\mathrm{S}$ & $\mathrm{S}$ & $\mathrm{S}$ & $\mathrm{S}$ & $S$ & $\mathrm{~S}$ \\
\hline Rádio Comunicador & $\mathrm{N}$ & $\mathrm{N}$ & $\mathrm{N}$ & $\mathrm{N}$ & $\mathrm{N}$ & $\mathrm{N}$ & $\mathrm{N}$ & $\mathrm{N}$ \\
\hline Identificador de Coletivos & $\mathrm{S}$ & $\mathrm{S}$ & $\mathrm{S}$ & $\mathrm{S}$ & $\mathrm{S}$ & $\mathrm{S}$ & $\mathrm{S}$ & $\mathrm{S}$ \\
\hline Registrador de passagem & $\mathrm{S}$ & $S$ & $S$ & $\mathrm{~S}$ & $\mathrm{~S}$ & $\mathrm{~S}$ & $S$ & $\mathrm{~S}$ \\
\hline $\begin{array}{l}\text { Registrador de coletivos } \\
\text { por satélite }\end{array}$ & $\mathrm{S}$ & S & S & $\mathrm{S}$ & $S$ & $\mathrm{~S}$ & $\mathrm{~S}$ & $\mathrm{~S}$ \\
\hline $\begin{array}{l}\text { Registrador do } \\
\text { comportamento do } \\
\text { condutor e do veículo }\end{array}$ & $\mathrm{S}$ & S & S & $\mathrm{S}$ & S & S & S & $\mathrm{S}$ \\
\hline Registrador de Viagens & $\mathrm{S}$ & $S$ & $S$ & $\mathrm{~S}$ & $S$ & $\mathrm{~S}$ & $S$ & $S$ \\
\hline Sinalização Braile & $\mathrm{S}$ & $S$ & $S$ & $\mathrm{~S}$ & $S$ & $\mathrm{~S}$ & $\mathrm{~S}$ & $\mathrm{~S}$ \\
\hline Biometria Facial & $\mathrm{S}$ & $\mathrm{S}$ & $S$ & $\mathrm{~S}$ & $S$ & $\mathrm{~S}$ & $\mathrm{~S}$ & $\mathrm{~S}$ \\
\hline Comunicador-usuário & $\mathrm{S}$ & $S$ & $S$ & $\mathrm{~S}$ & $S$ & $\mathrm{~S}$ & $\mathrm{~S}$ & $\mathrm{~S}$ \\
\hline Acionador de semáforo & $\mathrm{N}$ & $\mathrm{N}$ & $\mathrm{N}$ & $\mathrm{N}$ & $\mathrm{N}$ & $\mathrm{N}$ & $\mathrm{N}$ & $\mathrm{N}$ \\
\hline Vigiador de vias e interior & $\mathrm{S}$ & $\mathrm{S}$ & $\mathrm{S}$ & $\mathrm{S}$ & $\mathrm{S}$ & $\mathrm{S}$ & $\mathrm{S}$ & $\mathrm{S}$ \\
\hline $\begin{array}{l}\text { Global Positioning System } \\
\text { (GPS) }\end{array}$ & $\mathrm{S}$ & $\mathrm{S}$ & $S$ & $\mathrm{~S}$ & $S$ & $\mathrm{~S}$ & $S$ & S \\
\hline Big Data & $\mathrm{S}$ & $\mathrm{S}$ & $\mathrm{S}$ & $\mathrm{S}$ & $S$ & $\mathrm{~S}$ & $S$ & $\mathrm{~S}$ \\
\hline Data Mining & $\mathrm{S}$ & $S$ & $S$ & $\mathrm{~S}$ & $S$ & $\mathrm{~S}$ & $\mathrm{~S}$ & $S$ \\
\hline Cloud Computing & $\mathrm{S}$ & $S$ & $\mathrm{~S}$ & $\mathrm{~S}$ & $S$ & $\mathrm{~S}$ & $\mathrm{~S}$ & $\mathrm{~S}$ \\
\hline Internet das Coisas & $\mathrm{S}$ & $\mathrm{S}$ & $\mathrm{S}$ & $\mathrm{S}$ & $\mathrm{S}$ & $\mathrm{S}$ & $\mathrm{S}$ & $\mathrm{S}$ \\
\hline Internet dos Serviços & $\mathrm{S}$ & $S$ & $\mathrm{~S}$ & $\mathrm{~S}$ & $S$ & $\mathrm{~S}$ & $\mathrm{~S}$ & $\mathrm{~S}$ \\
\hline Crowdsourcing & $\mathrm{S}$ & $\mathrm{S}$ & $\mathrm{S}$ & $\mathrm{S}$ & $\mathrm{S}$ & $\mathrm{S}$ & $\mathrm{S}$ & $\mathrm{S}$ \\
\hline Blockchain & $\mathrm{NE}$ & $\mathrm{NE}$ & $\mathrm{NE}$ & $\mathrm{NE}$ & $\mathrm{NE}$ & $\mathrm{NE}$ & $\mathrm{NE}$ & $\mathrm{NE}$ \\
\hline Segurança Cibernética & $\mathrm{S}$ & $\mathrm{S}$ & $\mathrm{S}$ & $\mathrm{S}$ & $\mathrm{S}$ & $\mathrm{S}$ & $\mathrm{S}$ & $\mathrm{S}$ \\
\hline Sistemas integrados & $S$ & $S$ & $\mathrm{~S}$ & $\mathrm{~S}$ & $S$ & $\mathrm{~S}$ & $S$ & $\mathrm{~S}$ \\
\hline Sanitização & $\mathrm{S}$ & $\mathrm{S}$ & $\mathrm{S}$ & $\mathrm{S}$ & $\mathrm{S}$ & $\mathrm{S}$ & $\mathrm{S}$ & $\mathrm{S}$ \\
\hline Total de Tecnologias & 29 & 29 & 29 & 29 & 29 & 29 & 29 & 29 \\
\hline Tipos de Energia & Diesel & Gas & Bateria & Elétrica & Elétrica & Elétrica & Elétrica & Elétrica \\
\hline Fontes de energia & NR & $\mathrm{R}$ & $\mathrm{R}$ & $\mathrm{R}$ & $\mathrm{R}$ & $\mathrm{R}$ & $\mathrm{R}$ & $\mathrm{R}$ \\
\hline
\end{tabular}

Fonte: Elaborado pelos autores (2021).

Na Figura 4 é apresentada a concentração das tecnologias e fontes de energia empregadas no transporte coletivo de transporte urbano de massa de Paris. 
Figura 4. Concentração de Tecnologias Inteligentes e Fontes de Energia para o Transporte de Massa da Cidade de Paris.

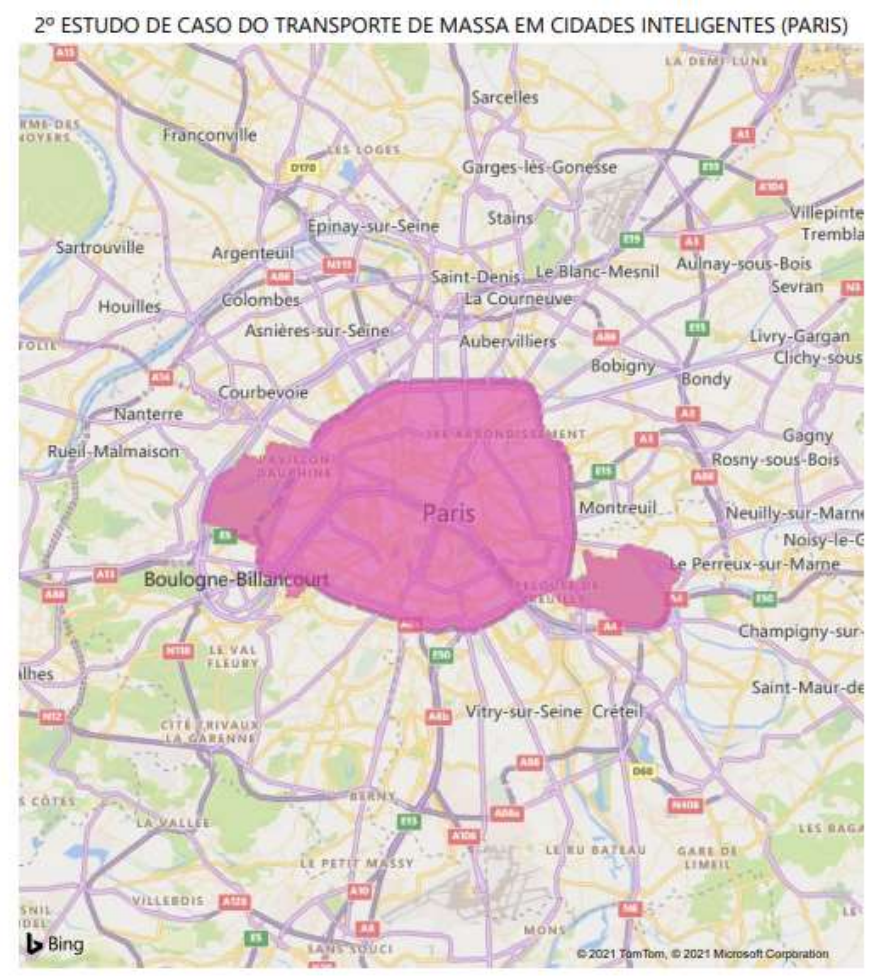

CONCENTRAÇĀO DAS TECNOLOGIAS INTELIGENTES NO TRANSPORTE DE MASSA

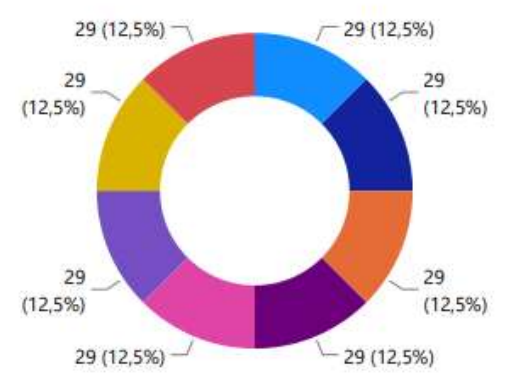

MODOS DE TRANSPORTE -METRÓ

- ÓNIBUS À BATERIA

- ÓNIBUS À DIESEL

- ÓNIBUs à gás

- TREM METROPOLITANo

- TREM URBANO

- TRÓLEBUS

๑VLT

\section{CONCENTRAÇĀO DAS FONTES DE ENERGIA NO TRANSPORTE DE MASSA}

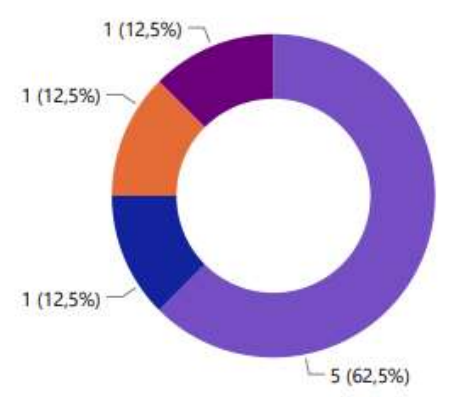

FONTES DE ENERGIA

- ELETRIFICADA

- bateria

- DIESEL

- Gás

Fonte: Elaborado pelos autores (2021).

Pode-se observar na Figura 4 que a concentração das tecnologias inteligentes no transporte de massa está equilibrada em quantidade e percentuais nos modos de transporte. Dessa forma, torna-se uma estratégia para melhorar a comunicação das tecnologias na mobilidade urbana da cidade inteligente de Paris. Sobre a concentração das fontes de energia no transporte de massa, é feito maior uso de energias renováveis, buscando a sustentabilidade através da redução da poluição atmosférica e sonora nas cidades inteligentes. Paris tem como meta a redução gradual ou a proibição de todos os tipos de veículos a diesel a partir de 2025. A cidade tem como "poderes parciais" da prefeitura a operação, a definição e aplicação das políticas de transporte público e como poderes limitados o controle orçamentário e de receita e visão definida no transporte público (C40 CITIES, 2021).

A vulnerabilidade do seu sistema de transporte de massa está na adesão da fonte de energia elétrica para ônibus. Suas tecnologias inteligentes e fontes de energia de transporte de massa são similares às da cidade inteligente de Madri.

\subsubsection{Caracterização Estudo de Caso 3 (Cidade Inteligente de São Paulo)}

São Paulo é a maior cidade brasileira, e a maior a nível sul-americano. A atividade econômica tem como base o setor de serviços, o comércio e a indústria. Geograficamente está localizada na região sudeste do Brasil, próxima do maior porto brasileiro, localizado na cidade de Santos (Porto de Santos) (Prefeitura de São Paulo, 2020). Estrategicamente sua localização auxilia a capilaridade das redes de transporte rodoviário, ferroviário e aeroviários de todo o país. Sua região metropolitana tem 21.571.281 habitantes (ver Tabela 1) (Prefeitura de São Paulo, 2020).

Os municípios de Guarulhos, Itaquaquecetuba, Poá, Ferraz de Vasconcelos, Mauá, Santo André, São Bernardo do Campo, São Caetano do Sul, Diadema, São Vicente, Mongaguá, Itanhaém, Embu-Guaçu, Itapecerica da Serra, Embu, Taboão de Serra, Cotia, Osasco, Barueri, Santana do Parnaíba, Cajamar, Caieiras e Mairiporã que se tornam cidades-dormitório, compreendendo vinte e três municípios limítrofes (Prefeitura de São Paulo, 2020). 
O sistema de transporte público de massa de São Paulo possui o metrô, o monotrilho, o trem, o ônibus urbano e interurbano, o micro-ônibus e as vans, e tem explorada a concessão do transporte público/privado. O sistema de transporte público de São Paulo é gerenciado pelo conjunto das seguintes empresas consorciadas: Companhia do Metropolitano de São Paulo (Metrô de São Paulo), para o sistema de metrô e do monotrilho; Companhia Paulista de Trens Metropolitanos (CPTM), para o sistema de trens urbanos e metropolitanos; Empresa Metropolitana de Transportes Urbanos de São Paulo (EMTU/SP), para o sistema de linhas de ônibus, micro-ônibus de São Paulo para metropolitanos; SPTrans, para o sistema de linhas de ônibus dos subúrbios do município e as Cooperativas de micro-ônibus e vans, que organiza os serviços de transporte público regular de passageiros, à procura e escolar.

O sistema de bilhetagem para o transporte público de massa é composto por vários tipos de bilhetes como:

- Bilhete único: cartão para a compra de crédito Comum, Estudante e Vale-Transporte, para uso nos ônibus, microônibus e trens do Metrô e da CPTM.

- Bilhete comum: é utilizado nos ônibus, micro-ônibus, metrôs e trens.

- Bilhete estudante/professor: é utilizado nos ônibus municipais, metrôs e trens.

- Bilhete especial: para uso de pessoas idosas, com deficiência, obesas e gestantes.

A Tabela 4 apresenta as tecnologias empregadas no transporte coletivo de transporte urbano de massa em São Paulo. 
Research, Society and Development, v. 10, n. 13, e37101320634, 2021

(CC BY 4.0) | ISSN 2525-3409 | DOI: http://dx.doi.org/10.33448/rsd-v10i13.20634

Tabela 4. Composição do Sistema de Transporte Urbano de Massa de São Paulo.

\begin{tabular}{|c|c|c|c|c|c|c|c|c|}
\hline \multirow{2}{*}{$\frac{\text { São Paulo }}{\text { Tecnologia }}$} & \multicolumn{8}{|c|}{ Modos de Transporte Coletivo Público e Urbano de Massa } \\
\hline & $\begin{array}{l}\text { Ônibus a } \\
\text { Diesel }\end{array}$ & $\begin{array}{l}\text { Ônibus } \\
\text { a Gás }\end{array}$ & $\begin{array}{c}\text { Ônibus a } \\
\text { Bateria }\end{array}$ & Trólebus & VLT & Metrô & $\begin{array}{c}\text { Trem } \\
\text { Urbano }\end{array}$ & $\begin{array}{c}\text { Trem } \\
\text { Suburbano }\end{array}$ \\
\hline ITS & $\mathrm{S}$ & $\mathrm{S}$ & $\mathrm{S}$ & $\mathrm{S}$ & $*$ & $\mathrm{~S}$ & S & $\mathrm{S}$ \\
\hline Ar condicionado & $\mathrm{S}$ & $\mathrm{S}$ & $\mathrm{S}$ & $\mathrm{S}$ & & $\mathrm{S}$ & $\mathrm{S}$ & $\mathrm{S}$ \\
\hline Internet 4G-usuário & $\mathrm{N}$ & $\mathrm{N}$ & $\mathrm{N}$ & $\mathrm{N}$ & * & $\mathrm{N}$ & $\mathrm{N}$ & $\mathrm{N}$ \\
\hline Internet 5G-usuário & $\mathrm{N}$ & $\mathrm{N}$ & $\mathrm{N}$ & $\mathrm{N}$ & $*$ & $\mathrm{~N}$ & $\mathrm{~N}$ & $\mathrm{~N}$ \\
\hline $\begin{array}{l}\text { Painéis de Informação de } \\
\text { horários e rotas }\end{array}$ & $\mathrm{S}$ & S & S & S & $*$ & $\mathrm{~S}$ & S & $\mathrm{S}$ \\
\hline Aplicativos-usuário & $\mathrm{S}$ & $\mathrm{S}$ & $\mathrm{S}$ & $\mathrm{S}$ & $*$ & $\mathrm{~S}$ & $\mathrm{~S}$ & $\mathrm{~S}$ \\
\hline $\begin{array}{l}\text { Registrador Mecânico de } \\
\text { passageiros }\end{array}$ & $S$ & S & S & S & $*$ & $S$ & S & $\mathrm{S}$ \\
\hline $\begin{array}{l}\text { Registrador Eletrônico de } \\
\text { embarques e desembarques }\end{array}$ & $\mathrm{N}$ & $\mathrm{N}$ & $\mathrm{N}$ & $\mathrm{N}$ & $*$ & $\mathrm{~N}$ & $\mathrm{~N}$ & $\mathrm{~N}$ \\
\hline $\begin{array}{l}\text { Emissor de comprovante } \\
\text { de pagamento }\end{array}$ & $\mathrm{S}$ & S & S & S & $*$ & $S$ & S & $\mathrm{S}$ \\
\hline Bilhete magnético & $\mathrm{S}$ & $\mathrm{S}$ & S & $\mathrm{S}$ & $*$ & $\mathrm{~S}$ & $\mathrm{~S}$ & $\mathrm{~S}$ \\
\hline Cartão inteligente & $\mathrm{N}$ & $\mathrm{N}$ & $\mathrm{N}$ & $\mathrm{N}$ & $*$ & $\mathrm{~N}$ & $\mathrm{~N}$ & $\mathrm{~N}$ \\
\hline Rádio Comunicador & $\mathrm{S}$ & $S$ & $S$ & $S$ & $*$ & $\mathrm{~S}$ & $\mathrm{~S}$ & $S$ \\
\hline Identificador de Coletivos & $\mathrm{S}$ & $S$ & $S$ & $S$ & $*$ & $\mathrm{~S}$ & $S$ & $\mathrm{~S}$ \\
\hline Registrador de passagem & $\mathrm{S}$ & $S$ & $S$ & $S$ & $*$ & $S$ & $S$ & $\mathrm{~S}$ \\
\hline $\begin{array}{l}\text { Registrador de coletivos } \\
\text { por satélite }\end{array}$ & $\mathrm{S}$ & $S$ & S & $S$ & $*$ & $\mathrm{~S}$ & $S$ & $\mathrm{~S}$ \\
\hline $\begin{array}{l}\text { Registrador do } \\
\text { comportamento do } \\
\text { condutor e do veículo }\end{array}$ & $\mathrm{S}$ & S & S & S & $*$ & $\mathrm{~S}$ & S & $\mathrm{S}$ \\
\hline Registrador de Viagens & $\mathrm{S}$ & $\mathrm{S}$ & $\mathrm{S}$ & $\mathrm{S}$ & $*$ & $\mathrm{~S}$ & $\mathrm{~S}$ & $\mathrm{~S}$ \\
\hline Sinalização Braile & $\mathrm{S}$ & $\mathrm{S}$ & $\mathrm{S}$ & $\mathrm{S}$ & $*$ & $\mathrm{~S}$ & $\mathrm{~S}$ & $\mathrm{~S}$ \\
\hline Biometria Facial & $\mathrm{S}$ & $S$ & $\mathrm{~S}$ & $\mathrm{~S}$ & $*$ & $\mathrm{~S}$ & $\mathrm{~S}$ & $\mathrm{~S}$ \\
\hline Comunicador-usuário & $\mathrm{N}$ & $\mathrm{N}$ & $\mathrm{N}$ & $\mathrm{N}$ & $*$ & $\mathrm{~N}$ & $\mathrm{~N}$ & $\mathrm{~N}$ \\
\hline Acionador de semáforo & $\mathrm{S}$ & $\mathrm{S}$ & $\mathrm{S}$ & $\mathrm{S}$ & $*$ & $\mathrm{~S}$ & $\mathrm{~S}$ & $\mathrm{~S}$ \\
\hline Vigiador de vias e interior & $\mathrm{S}$ & $\mathrm{S}$ & S & $\mathrm{S}$ & $*$ & $\mathrm{~S}$ & $\mathrm{~S}$ & $\mathrm{~S}$ \\
\hline $\begin{array}{l}\text { Global Positioning System } \\
\text { (GPS) }\end{array}$ & $\mathrm{S}$ & S & $\mathrm{S}$ & $\mathrm{S}$ & $*$ & $\mathrm{~S}$ & S & S \\
\hline Big Data & $\mathrm{S}$ & $\mathrm{S}$ & $\mathrm{S}$ & $\mathrm{N}$ & $*$ & $\mathrm{~S}$ & $\mathrm{~S}$ & $\mathrm{~S}$ \\
\hline Data Mining & $\mathrm{N}$ & $\mathrm{N}$ & $\mathrm{N}$ & $\mathrm{N}$ & $*$ & $\mathrm{~S}$ & $\mathrm{~N}$ & $\mathrm{~N}$ \\
\hline Cloud Computing & $\mathrm{N}$ & $\mathrm{N}$ & $\mathrm{N}$ & $\mathrm{N}$ & $*$ & $\mathrm{~S}$ & $\mathrm{~S}$ & $\mathrm{~S}$ \\
\hline Internet das Coisas & $\mathrm{S}$ & $\mathrm{S}$ & $\mathrm{S}$ & $\mathrm{N}$ & $*$ & $\mathrm{~S}$ & $\mathrm{~N}$ & $\mathrm{~N}$ \\
\hline Internet dos Serviços & $\mathrm{S}$ & $\mathrm{S}$ & $\mathrm{S}$ & $\mathrm{N}$ & $*$ & $\mathrm{~S}$ & $\mathrm{~N}$ & $\mathrm{~N}$ \\
\hline Crowdsourcing & $\mathrm{N}$ & $\mathrm{N}$ & $\mathrm{N}$ & $\mathrm{N}$ & $*$ & $\mathrm{~S}$ & $\mathrm{~N}$ & $\mathrm{~N}$ \\
\hline Blockchain & NE & $\mathrm{NE}$ & $\mathrm{NE}$ & NE & NE & $\mathrm{NE}$ & NE & $\mathrm{NE}$ \\
\hline Segurança Cibernética & $\mathrm{S}$ & $\mathrm{S}$ & $S$ & $\mathrm{~N}$ & $*$ & $\mathrm{~S}$ & $S$ & $\mathrm{~S}$ \\
\hline Sistemas integrados & $\mathrm{S}$ & $S$ & $\mathrm{~S}$ & $\mathrm{~N}$ & $*$ & $\mathrm{~S}$ & $\mathrm{~S}$ & $\mathrm{~S}$ \\
\hline Sanitização & $\mathrm{S}$ & $\mathrm{S}$ & $\mathrm{S}$ & $\mathrm{S}$ & $*$ & $\mathrm{~S}$ & $\mathrm{~S}$ & $\mathrm{~S}$ \\
\hline Total de Tecnologias & 24 & 24 & 24 & 19 & $*$ & 27 & 20 & 25 \\
\hline Tipos de Energia & Diesel & Gas & Bateria & Elétrica & $*$ & Elétrica & Elétrica & Elétrica \\
\hline Fontes de energia & NR & $\mathrm{R}$ & $\mathrm{R}$ & $\mathrm{R}$ & $*$ & $\mathrm{R}$ & $\mathrm{R}$ & $\mathrm{R}$ \\
\hline
\end{tabular}

Fonte: Elaborado pelos autores (2021).

Na Figura 5 é apresentada a concentração das tecnologias e fontes de energia empregadas no transporte coletivo de transporte urbano de massa de São Paulo. 
Figura 5. Concentração de Tecnologias Inteligentes e Fontes de Energia para o Transporte de Massa da Cidade de São Paulo.

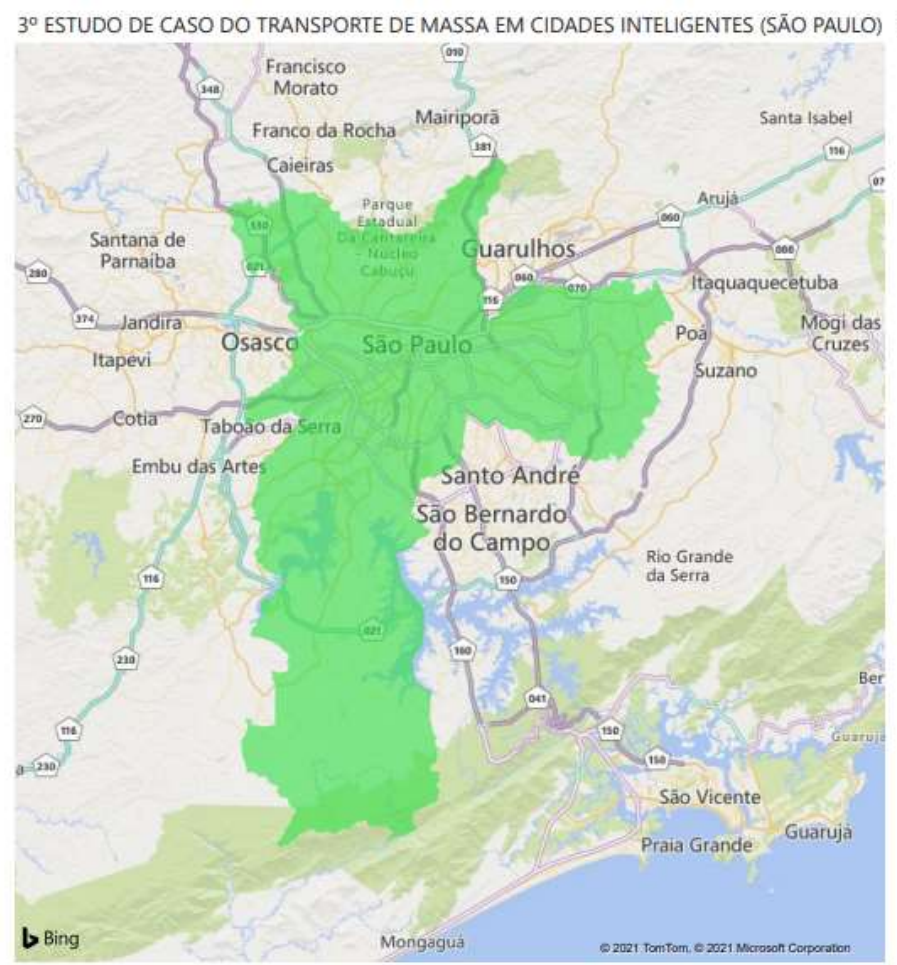

CONCENTRAÇĀO DAS TECNOLOGIAS INTELIGENTES NO TRANSPORTE DE MASSA

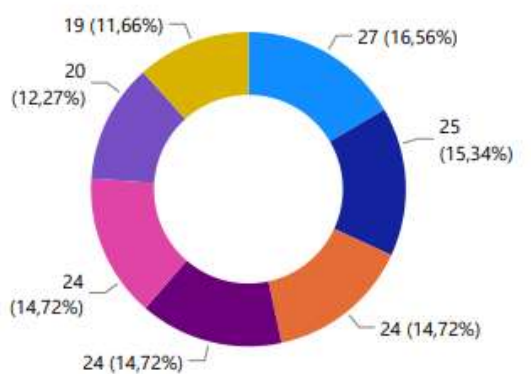

- TREM METROPOLITANO

- ÔNIBUS À BATERIA

-ÓNIBUS À DIESEL

- ÓNIBUS Á GÁs

- TREM URBANO

$24(14,72 \%)$

etrólebus

CONCENTRAÇÃO DAS FONTES DE ENERGIA NO TRANSPORTE DE MASSA

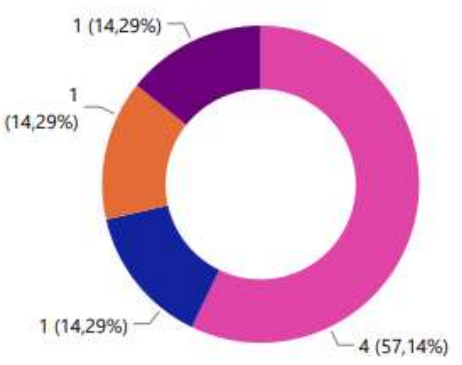

FONTES DE ENERGIA

- ELETRIFICADA

- BATERIA

- DIESEL

- GÁs

Fonte: Elaborado pelos autores (2021).

Pode-se observar na Figura 5 que a concentração das tecnologias inteligentes no transporte de massa está desequilibrada em quantidade e percentuais nos modos de transporte. O modo de transporte de massa "metrô" concentra o maior número de tecnologias inteligentes, de forma que é importante que haja políticas de equidade tecnológica para se alcançar a distribuição das tecnologias de maneira mais uniforme entre todos os modos de transporte urbano levantados, melhorando o fluxo da mobilidade inteligente da cidade inteligente de São Paulo. Sobre a concentração das fontes de energia no transporte de massa, conforme se vê na Figura 4, há o uso maior de energias renováveis buscando a sustentabilidade através da redução da poluição atmosférica e sonora nas cidades inteligentes. São Paulo tem como meta a redução gradual ou a proibição de todos os tipos de veículos a diesel a partir de 2025 tem como "poderes parciais" da prefeitura a operação, a definição e aplicação das políticas, o controle orçamentário e de receita e visão definida no transporte público (C40 CITIES, 2021).

A vulnerabilidade do seu sistema de transporte de massa está na adesão da fonte de energia elétrica para ônibus e a falta de um sistema de Veículos Leve Sobre os Trilhos (VLT) e na adesão da fonte de energia elétrica para ônibus. Suas tecnologias inteligentes e fontes de energia de transporte de massa são similares ás da cidade inteligente de Buenos Aires. A falta de implantação da internet $4 \mathrm{G}$ e $5 \mathrm{G}$ reduz consideravelmente a velocidade de transmissão dos dados nas tecnologias inteligentes disponíveis no transporte de massa levantado, limitando a capilaridade das informações e influenciando nos resultados dos fluxos de mobilidade urbana, mobilidade inteligente e sustentável na cidade inteligente.

\subsubsection{Caracterização Estudo de Caso 4 (Cidade Inteligente de Buenos Aires)}

Buenos Aires é a maior cidade argentina, e a terceira a nível sul-americano. A atividade econômica tem como base o setor de serviços, o comércio e a indústria. Geograficamente está localizada na região sudeste da Argentina, na costa ocidental 
do estuário do Rio da Prata. A cidade de Buenos Aires tem o maior porto de país (Porto de Buenos Aires), que concentra 40\% do total do comércio exterior movimentado pelos país. Estrategicamente, sua localização auxilia a capilaridade das redes de transporte rodoviário, ferroviário e aeroviários de todo o país (Buenos Aires Ciudad BA, 2020; Instituto Nacional de Estadística y Censos República Argentina, 2020). Sua região metropolitana tem 15.594.428 habitantes (Tabela 1) (Buenos Aires Ciudad BA, 2020).

Os municípios de Avellaneda, Quilmes, General San Martin, Vicente Lopez, Colinas de Zamora, La Matanza, San Isidro, Almirante Brown, Berazategui, Florencio Varela, Idiota, Hurlingham, San Fernando, Ituzaingo, Tigre, Ezeiza, Esteban Echeverria, San Miguel e Malvinas Argentinas são cidades-dormitório, totalizando vinte e um municípios limítrofes (Buenos Aires Ciudad BA, 2020). O sistema de transporte público de massa de Buenos Aires possui o metrô, o trem, o ônibus urbano e interurbano e é explorada a concessão do transporte público/privado.

O sistema de transporte público de Buenos Aires é gerenciado pelo conjunto das seguintes empresas consorciadas: Subterráneos de Buenos Aires (SBASE ou Subte e Metrovías) e Premetro, para o sistema de metrô; as companhias Trens argentinos, Ferrovias e Metrovías, para o sistema de trens urbanos e metropolitanos; Plaza e Line 132, Metrobús, para o sistema de Bus Rapid Transit (BRT); e o Ecobici para o sistema de uso de bicicletas gratuitas. O sistema de bilhetagem para o transporte público de massa é único, como pode ser observado a seguir:

- Bilhete único: O Sistema Único de Boleto Electrónico (Subet), ou Cartão MONEDERO, é um sistema pré-pago que permite aos usuários pagar viagens de ônibus, metrô, pedágios e trens com um único cartão.

A Tabela 5 apresenta as tecnologias empregadas no transporte coletivo de transporte urbano de massa em Buenos Aires. 
Tabela 5. Composição do Sistema de Transporte Urbano de Massa de Buenos Aires.

\begin{tabular}{|c|c|c|c|c|c|c|c|c|}
\hline \multirow{2}{*}{$\begin{array}{c}\text { Buenos Aires } \\
\text { Tecnologia }\end{array}$} & \multicolumn{8}{|c|}{ Modos de Transporte Coletivo Público e Urbano de Massa } \\
\hline & $\begin{array}{l}\text { Ônibus a } \\
\text { Diesel }\end{array}$ & $\begin{array}{l}\text { Ônibus } \\
\text { a Gás }\end{array}$ & $\begin{array}{c}\text { Ônibus a } \\
\text { Bateria }\end{array}$ & Trólebus & VLT & Metrô & $\begin{array}{c}\text { Trem } \\
\text { Urbano }\end{array}$ & $\begin{array}{c}\text { Trem } \\
\text { Suburbano }\end{array}$ \\
\hline ITS & $\mathrm{S}$ & $\mathrm{S}$ & $\mathrm{S}$ & $\mathrm{S}$ & $*$ & $\mathrm{~S}$ & $\mathrm{~S}$ & $\mathrm{~S}$ \\
\hline Ar condicionado & $\mathrm{S}$ & $\mathrm{S}$ & S & $\mathrm{S}$ & $*$ & $\mathrm{~S}$ & $\mathrm{~S}$ & $\mathrm{~S}$ \\
\hline Internet 4G-usuário & $\mathrm{S}$ & $\mathrm{S}$ & $\mathrm{S}$ & $\mathrm{S}$ & $*$ & $\mathrm{~S}$ & $\mathrm{~S}$ & $\mathrm{~S}$ \\
\hline Internet 5G-usuário & $\mathrm{N}$ & $\mathrm{N}$ & $\mathrm{N}$ & $\mathrm{N}$ & $*$ & $\mathrm{~N}$ & $\mathrm{~N}$ & $\mathrm{~N}$ \\
\hline $\begin{array}{l}\text { Painéis de Informação de } \\
\text { horários e rotas }\end{array}$ & $\mathrm{S}$ & $\mathrm{S}$ & $\mathrm{S}$ & $\mathrm{S}$ & $*$ & $\mathrm{~S}$ & $\mathrm{~S}$ & $\mathrm{~S}$ \\
\hline Aplicativos-usuário & $\mathrm{S}$ & $\mathrm{S}$ & $S$ & $\mathrm{~S}$ & $*$ & $\mathrm{~S}$ & $\mathrm{~S}$ & $\mathrm{~S}$ \\
\hline $\begin{array}{l}\text { Registrador Mecânico de } \\
\text { passageiros }\end{array}$ & $\mathrm{S}$ & $\mathrm{S}$ & S & $\mathrm{S}$ & $*$ & $\mathrm{~S}$ & $S$ & S \\
\hline $\begin{array}{l}\text { Registrador Eletrônico de } \\
\text { embarques e desembarques }\end{array}$ & $\mathrm{S}$ & $\mathrm{S}$ & $\mathrm{S}$ & $S$ & $*$ & $\mathrm{~S}$ & $S$ & $\mathrm{~S}$ \\
\hline $\begin{array}{l}\text { Emissor de comprovante } \\
\text { de pagamento }\end{array}$ & $S$ & S & S & S & $*$ & S & S & S \\
\hline Bilhete magnético & $S$ & $S$ & $S$ & $S$ & $*$ & $S$ & $S$ & $S$ \\
\hline Cartão inteligente & $\mathrm{S}$ & $\mathrm{S}$ & $S$ & $\mathrm{~S}$ & $*$ & $\mathrm{~S}$ & $\mathrm{~S}$ & $\mathrm{~S}$ \\
\hline Rádio Comunicador & $S$ & $S$ & $S$ & $S$ & $*$ & $S$ & $S$ & $S$ \\
\hline Identificador de Coletivos & $S$ & $S$ & $S$ & $S$ & $*$ & $S$ & $\mathrm{~S}$ & $S$ \\
\hline Registrador de passagem & $\mathrm{S}$ & $S$ & $S$ & $S$ & $*$ & $\mathrm{~S}$ & $S$ & $S$ \\
\hline $\begin{array}{l}\text { Registrador de coletivos } \\
\text { por satélite }\end{array}$ & $\mathrm{S}$ & $\mathrm{S}$ & $\mathrm{S}$ & $\mathrm{S}$ & $*$ & $\mathrm{~S}$ & $\mathrm{~S}$ & $\mathrm{~S}$ \\
\hline $\begin{array}{l}\text { Registrador do } \\
\text { comportamento do } \\
\text { condutor e do veículo }\end{array}$ & $\mathrm{NE}$ & $\mathrm{NE}$ & NE & NE & $*$ & $\mathrm{NE}$ & $\mathrm{NE}$ & $\mathrm{NE}$ \\
\hline Registrador de Viagens & $\mathrm{S}$ & $\mathrm{S}$ & $\mathrm{S}$ & $\mathrm{S}$ & $*$ & $\mathrm{~S}$ & $\mathrm{~S}$ & $\mathrm{~S}$ \\
\hline Sinalização Braile & $\mathrm{NE}$ & $\mathrm{NE}$ & $\mathrm{NE}$ & $\mathrm{NE}$ & $*$ & $\mathrm{~S}$ & $\mathrm{NE}$ & $\mathrm{NE}$ \\
\hline Biometria Facial & $\mathrm{NE}$ & $\mathrm{NE}$ & $\mathrm{NE}$ & $\mathrm{NE}$ & $*$ & $\mathrm{~S}$ & $\mathrm{NE}$ & $\mathrm{NE}$ \\
\hline Comunicador-usuário & $\mathrm{S}$ & $\mathrm{S}$ & $\mathrm{S}$ & $\mathrm{S}$ & $*$ & $\mathrm{~S}$ & $\mathrm{~S}$ & $\mathrm{~S}$ \\
\hline Acionador de semáforo & $\mathrm{NE}$ & $\mathrm{NE}$ & $\mathrm{NE}$ & $\mathrm{NE}$ & $*$ & $\mathrm{~S}$ & $\mathrm{NE}$ & $\mathrm{NE}$ \\
\hline Vigiador de vias e interior & $\mathrm{NE}$ & $\mathrm{NE}$ & $\mathrm{NE}$ & $\mathrm{NE}$ & $*$ & $S$ & $\mathrm{NE}$ & $\mathrm{NE}$ \\
\hline $\begin{array}{l}\text { Global Positioning System } \\
\text { (GPS) }\end{array}$ & $\mathrm{S}$ & $S$ & $S$ & $S$ & $*$ & $\mathrm{~S}$ & $S$ & $\mathrm{~S}$ \\
\hline Big Data & $\mathrm{NE}$ & $\mathrm{NE}$ & $\mathrm{NE}$ & $\mathrm{NE}$ & $*$ & $\mathrm{~S}$ & $\mathrm{NE}$ & $\mathrm{NE}$ \\
\hline Data Mining & $\mathrm{NE}$ & $\mathrm{NE}$ & $\mathrm{NE}$ & $\mathrm{NE}$ & $*$ & $S$ & $\mathrm{NE}$ & $\mathrm{NE}$ \\
\hline Cloud Computing & $\mathrm{NE}$ & $\mathrm{NE}$ & $\mathrm{NE}$ & $\mathrm{NE}$ & $*$ & $\mathrm{~S}$ & $\mathrm{NE}$ & $\mathrm{NE}$ \\
\hline Internet das Coisas & $\mathrm{NE}$ & $\mathrm{NE}$ & $\mathrm{NE}$ & $\mathrm{NE}$ & $*$ & $\mathrm{~S}$ & $\mathrm{NE}$ & $\mathrm{NE}$ \\
\hline Internet dos Serviços & $\mathrm{NE}$ & $\mathrm{NE}$ & $\mathrm{NE}$ & $\mathrm{NE}$ & $*$ & $S$ & $\mathrm{NE}$ & $\mathrm{NE}$ \\
\hline Crowdsourcing & $\mathrm{NE}$ & $\mathrm{NE}$ & $\mathrm{NE}$ & $\mathrm{NE}$ & $*$ & $S$ & $\mathrm{NE}$ & $\mathrm{NE}$ \\
\hline Blockchain & $\mathrm{NE}$ & $\mathrm{NE}$ & $\mathrm{NE}$ & $\mathrm{NE}$ & $*$ & $\mathrm{NE}$ & $\mathrm{NE}$ & $\mathrm{NE}$ \\
\hline Segurança Cibernética & $\mathrm{NE}$ & $\mathrm{NE}$ & $\mathrm{NE}$ & $\mathrm{NE}$ & $*$ & $\mathrm{~S}$ & $\mathrm{NE}$ & $\mathrm{NE}$ \\
\hline Sistemas integrado & $\mathrm{S}$ & $\mathrm{S}$ & $\mathrm{S}$ & $\mathrm{S}$ & $*$ & $\mathrm{~S}$ & $\mathrm{~S}$ & $\mathrm{~S}$ \\
\hline Sanitização & $\mathrm{NE}$ & $\mathrm{NE}$ & $\mathrm{NE}$ & $\mathrm{NE}$ & $*$ & $\mathrm{~S}$ & $\mathrm{NE}$ & $\mathrm{NE}$ \\
\hline Total de Tecnologias & 18 & 18 & 18 & 18 & $*$ & 30 & 18 & 18 \\
\hline Tipos de Energia & Diesel & Gas & Bateria & Elétrica & $*$ & Elétrica & Elétrica & Elétrica \\
\hline Fontes de energia & NR & $\mathrm{R}$ & $\mathrm{R}$ & $\mathrm{R}$ & $*$ & $\mathrm{R}$ & $\mathrm{R}$ & $\mathrm{R}$ \\
\hline
\end{tabular}

Fonte: Elaborado pelos autores (2021).

Na Figura 6 é apresentada a concentração das tecnologias e fontes de energia empregadas no transporte coletivo de transporte urbano de massa de Buenos Aires. 
Figura 6. Concentração de Tecnologias Inteligentes e Fontes de Energia para o Transporte de Massa da Cidade de Buenos Aires.

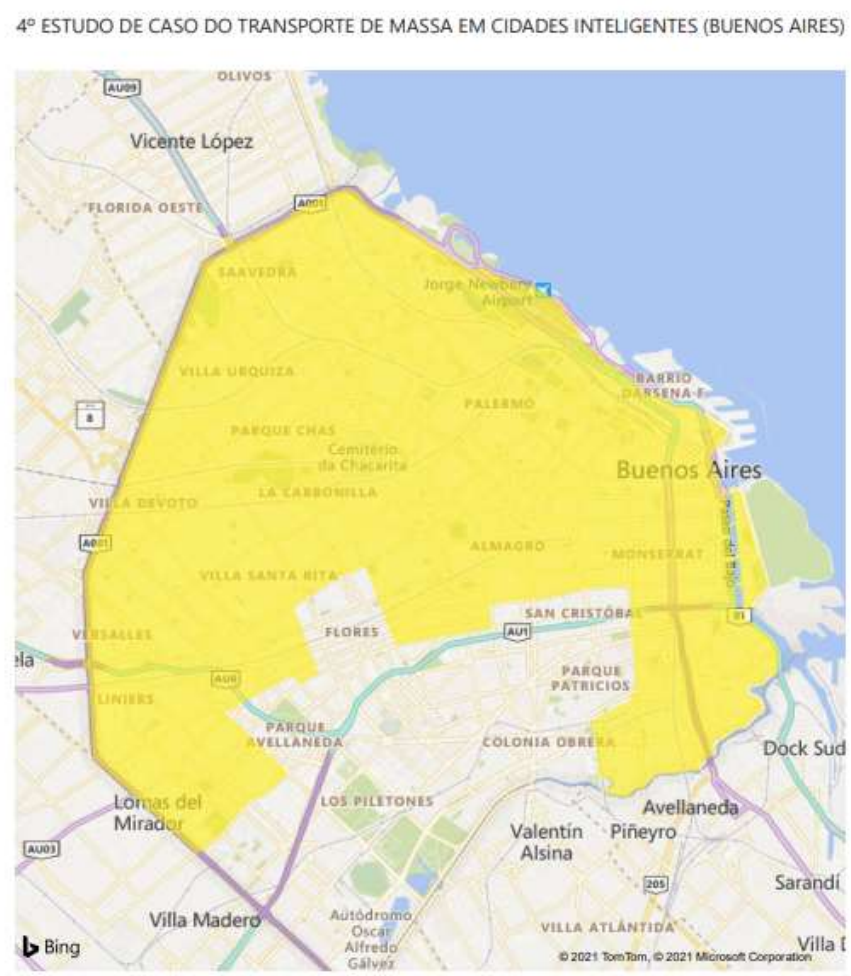
CONCENTRAÇĀO DAS TECNOLOGIAS INTELIGENTES NO TRANSPORTE DE MASSA

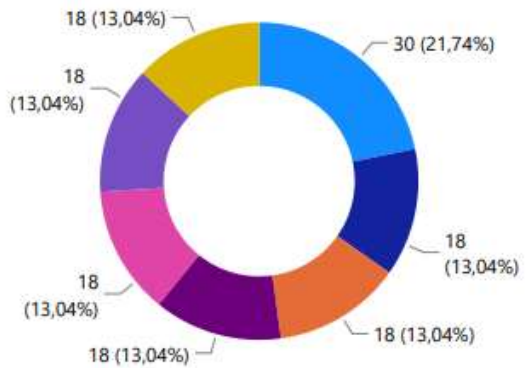

MODOS DE TRANSPORTE - METRÓ - ÓNIBUS À BATERIA -ÔNIBUS À DIESEL -ÓNIBUS Á GÁs - TREM METROPOLITANO - TREM URBANO - TRÓLEBUS - VLT

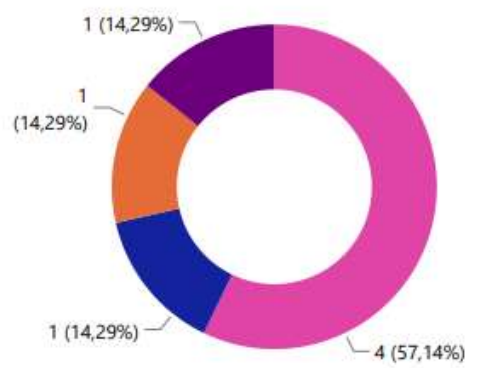

FONTES DE ENERGIA -eletrificada

- BATERIA - DIESEL - Gás

Fonte: Elaborado pelos autores (2021).

Pode-se observar na Figura 6, que a concentração das tecnologias inteligentes no transporte de massa está desequilibrada em relação à quantidade e aos percentuais nos modos de transporte, pois o modo de transporte de massa "metrô" concentra o maior número de tecnologias inteligentes. É importante que haja políticas de equidade tecnológica para se alcançar a distribuição das tecnologias de maneira mais uniforme entre todos os modos de transporte urbano levantados, melhorando a fluxo da mobilidade inteligente da cidade inteligente de Buenos Aires. A mobilidade inteligente compreende em uma série de medidas que visam simplificar a mobilidade dos usuários e que têm, como objetivo em comum, reduzir custos econômicos, ambientais e de tempo (Aletà et al., 2017).

Sobre a concentração das fontes de energia no transporte de massa, pode-se visualizar na Figura 5 o uso maior de energias renováveis, buscando a sustentabilidade através da redução da poluição atmosférica e sonora nas cidades inteligentes. Buenos Aires tem como meta a redução gradual ou a proibição de todos os tipos de veículos a diesel a partir de 2025 , e tem como "poderes parciais" da prefeitura a operação no transporte público, e tem como "poderes parciais" a definição e a aplicação das políticas, o controle orçamentário e de receita e a visão definida no transporte público (C40 CITIES, 2021).

A vulnerabilidade do seu sistema de transporte de massa está na adesão da fonte de energia elétrica para ônibus e a falta de um sistema de Veículos Leve Sobre os Trilhos (VLT). Suas tecnologias inteligentes e fontes de energia de transporte de massa são similares às da cidade inteligente de São Paulo. A falta de implantação da internet 4G e 5G reduz consideravelmente a velocidade de transmissão dos dados nas tecnologias inteligentes disponíveis no transporte de massa levantado, limitando a capilaridade das informações e influenciando nos resultados dos fluxos de mobilidade urbana, mobilidade inteligente e sustentável na cidade inteligente. 


\subsection{Classificação de sistema de transporte urbano de massa inteligente e sustentável}

Os dados coletados e cruzados pelas duas bases de dados das seções anteriores geraram a triangulação da terceira base. As informações das Figuras 3, 4, 5 e 6 são tratadas na Figura 7, cuja a proposta é a classificação dos sistemas de transporte urbano de massa por tecnologias inteligentes e sustentáveis.

Figura 7. Classificação do Transporte de Massa Urbano Inteligente e Sustentável em Quatro Cidades Inteligentes.

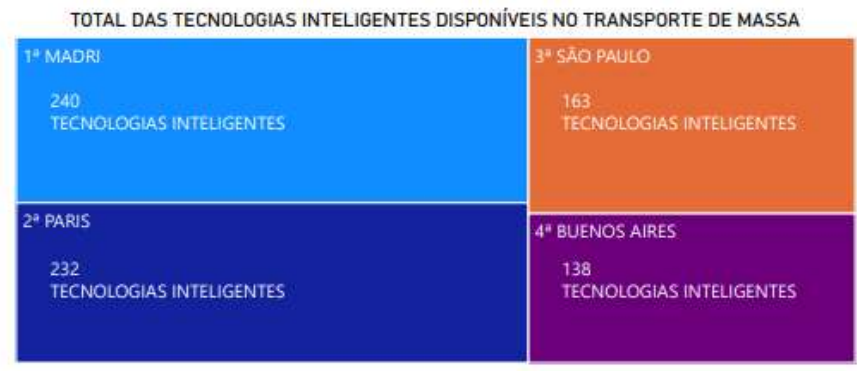

PERCENTUAL DAS TECNOLOGIAS INTELIGENTES DISPONIVEIS NO TRANSPORTE DE MASSA

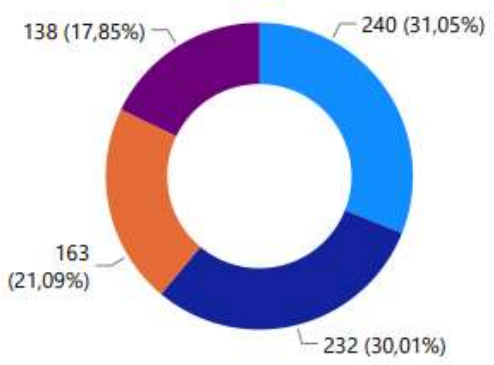

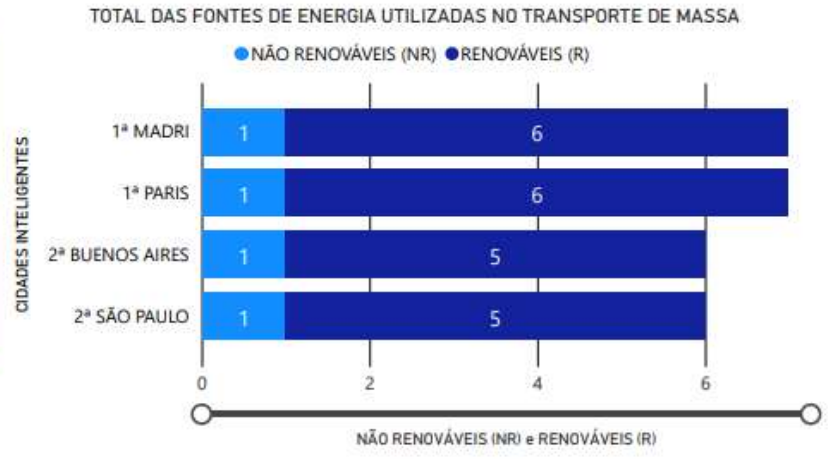

PERCENTUAL DAS FONTES DE ENERGIA UTILIZADAS NO TRANSPORTE DE MASSA - NÃO RENOVÁVEIS (NR) RENOVÁVEIS (R)

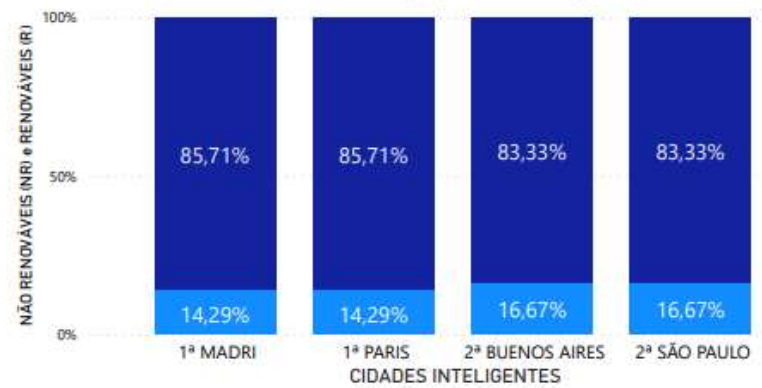

Fonte: Elaborado pelos autores (2021).

$\mathrm{Na}$ Figura 7, as cidades inteligentes podem ser classificadas pelo seu sistema de transporte de massa, considerando a quantidade de lacunas tecnológicas que possam existir entre elas. Por exemplo, a cidade de Madri ocupa a primeira colocação, com o total de 240 tecnologias inteligentes. Paris possui um total de 232 tecnologias inteligentes, ou seja, se comparada a Paris, Madri possui 8 tecnologias inteligentes a mais que a capital da França. A cidade de Paris ocupa a segunda colocação, contando com o total de 232 tecnologias inteligentes, 69 tecnologias a mais que a cidade de São Paulo, que possui um total de 163 tecnologias inteligentes. São Paulo ocupa a terceira colocação, contabilizando 163 tecnologias inteligentes, enquanto Buenos Aires possui 138 tecnologias inteligentes, 25 tecnologias a menos que São Paulo.

Questões como: a velocidade da internet, a disponibilidade de $4 \mathrm{G}$ ou $5 \mathrm{G}$ disponíveis no plano de mobilidade, a diversificação dos modos de transporte de massa ou mesmo a concentração de Intelligent Transportation Systems (ITS) ou tecnologias da quarta revolução industrial ou da Indústria 4.0 impactam no desempenho deste estudo de caso múltiplo. As tecnologias da Quarta Revolução Industrial e da marca da Indústria 4.0 como: a Internet das coisas ou IoT (Internet of Things), a Internet de Serviços ou IoS (Internet of Services), a Big Data, Computação em Nuvem (Cloud Computing), a segurança cibernética e a integração de sistemas (Sacomano et al, 2018), foram analisadas nos resultados da triangulação estudada. Identificou-se a aproximação ao tema Indústria 4.0 aplicada aos sistemas inteligentes e sustentáveis de transporte de massa nas quatro cidades inteligentes do estudo de caso múltiplo, nas Figuras 3, 4, 5 e 6. 
Sobre as tecnologias sustentáveis, conforme se vê na Figura 7, as quatro cidades inteligentes utilizam fontes de energia convencionais, porém, existe a lacuna tecnológica da transição dos ônibus a diesel para o ônibus elétrico, sendo uma tendência ligada à mudança dos sistemas de frotas de ônibus urbanos e metropolitanos, que concentram o maior volume de veículos para o transporte público (Holland et al., 2021)). Também na Figura 7, observa-se que existem iniciativas para modelos de fontes de energia renováveis como: a bateria, o gás e a já tradicionalmente utilizada eletricidade, para os modos de transporte de massa metrô, trens, VLT, trólebus. Esse fenômeno de fontes de energia renováveis no transporte de massa consolida a ideia de sustentabilidade no transporte público com os percentuais de Madri, Paris (85,71\%) e São Paulo e Buenos Aires (83,33\%).

O diesel ocupa uma parcela considerável de energia não renovável utilizada em Madri e Paris (14,29\%) e em São Paulo e Buenos Aires (16,67\%), se analisado com uma fonte de energia não renovável a todo o percentual do estudo de caso múltiplo do transporte de massa em cidades inteligentes.

Desta forma a proposição de um modelo de "Classificação dos Sistemas Inteligentes e Sustentáveis de Transporte de Massa em Cidades Inteligentes" pode ser pensando utilizando a classificação de maior concentração de tecnologias inteligentes disponíveis e maior concentração de fontes de energia renováveis disponíveis no sistema de transporte de massa em cidade inteligentes.

No contexto do estudo, pode-se então classificar Madri e Paris com 6 fontes de energia renováveis disponíveis para uso no transporte de massa, dando a essas cidades em primeiro lugar, e a São Paulo e Buenos Aires, em segundo lugar, com 5 fontes de energia renováveis disponíveis para o uso no transporte de massa.

Pode-se, portanto, concluir que quanto mais distante é o uso da diversificação dos modos de transporte urbano de massa, das tecnologias da quarta revolução industrial e da Industria 4.0 e da implantação de sistemas de fontes de energia renováveis, como os ônibus elétricos, mais distantes ficam as cidades de atender a complexidade da mobilidade urbana de massa. Em contrapartida, menos abertos estão esses sistemas de receberem mais dados de comunicação e tecnologias para mitigarem desafios e riscos nas cidades inteligentes, transformando essas informações estratégias em decisão em prol dos cidadãos.

A população necessita da melhoria contínua e atualizada das informações do transporte de massa pela governança das prefeituras e de sua a consciência das necessidades para oferecer os atributos do transporte como maior conforto, menor tempo de percurso, menor custo e maior acessibilidade, ajustando-se a demanda crescente das metrópoles (ver Tabela 1).

O principal desafio está em mudar o sistema produtivo e o fornecimento dessas tecnologias para o transporte público para a oferta de tecnologias inteligentes e renováveis para consumo das prefeituras, secretarias de transporte e mobilidade e dos cidadãos que utilizam esse sistema de transporte. Uma cidade inteligente é um sistema que se desenvolve pela influência de fatores externos, nesse caso, o elemento central é a participação direta dos atores e stakeholders locais no processo de pensar, definir, planejar e executar as transformações sociais, tecnológicas e urbanas nas cidades (Yigitcanlar et al., 2016).

Desta forma, essas tecnologias inteligentes e sustentáveis podem contribuir para a mitigação de riscos na mobilidade urbana em cidades inteligentes impactando em respostas instantâneas e simultâneas para a qualidade do transporte de massa.

\subsection{Análises do Estudo de Caso Múltiplo}

Madri, Paris, São Paulo e Buenos Aires trouxeram evidências para discussão da "Classificação de Sistema de Transporte Urbano de Massa Inteligente e Sustentável”, tais como: 
- Evidências pelo critério de Convergência dos Sistemas de Transporte Urbano de Massa: As cidades convergem para a prática de implantação de tecnologias disponíveis, portanto, elas têm em sua composição os Intelligent Transportation Systems (ITS) e as tecnologias de Indústria 4.0. Os ITS foram criados e evoluíram de 1970 a 2007 , trazendo aplicações das áreas como robótica, informática, telecomunicações e eletrônica para extração de dados, tratamento das informações sobre o trânsito e o tráfego (Nowacki, 2012). Atualmente, existem tecnologias de detecção, de comunicação sem fio, de tecnologia computacional, de análise do comportamento do usuário como parte da cadeia de transporte, dos modelos financeiros que influenciam os ITS, dos regulamentos, das políticas e dos padrões que afetam o futuro dos aplicativos ITS (Dimitrakopoulos et al., 2020).

- Evidências pelo critério de Divergência dos Sistemas de Transporte Urbano de Massa: Se analisados os quatro sistemas de transporte de massa nas cidades inteligentes pesquisadas é possível concluir que:

$\checkmark$ Os sistemas de transportes urbanos de massa têm uma classificação divergente, conforme a analise realizada, podendo os mesmos sistemas serem considerados mais ou menos diversificados; mais ou menos concentrados; mais ou menos disponíveis por suas tecnologias aplicadas; ou mais ou menos limpos; ou poluentes em suas fontes de energia (sendo elas renováveis ou não renováveis). Por exemplo, todas as cidades têm a dependência dos ônibus a diesel, trazendo uma prática de fonte de energia dieselizada, apesar de existirem alternativas de outros modos de transporte, como eletrificados, a gás ou bateria. Uma possibilidade a explorar são sistemas de transporte que utilizam biocombustíveis como etanol, porém a literatura é escassa. As demandas energéticas mundiais geram problemas como a degradação ambiental e a ineficiência energética no transporte público urbano, assim, busca-se as inovações tecnológicas como parte de uma solução ao transporte, portanto, a eficiência energética e a qualidade ambiental se tornam oportunidades para contribuir para a sustentabilidade energéticoambiental e econômica do transporte público urbano (Kneib \& Tedesco, 2010).

- Evidências pelo critério das Oportunidades dos Sistemas de Transporte Urbano de Massa: Atualmente, as cidades inteligentes exploram alternativas para responder ao crescimento da urbanização e suas consequências e desafios (como grande consumo de energia, alterações no clima e meio ambiente, tráfego e congestionamento, entre outros), servindo como tendência a tecnologia facilitadora e sendo alicerce para as cidades inteligentes (Alavi et al., 2018). Existem diversas estratégias de tecnologias em cidades inteligentes como: Sistemas Complexos e as Infraestruturas Digitais; a Big Data Analytics, IOT, Cloud Computing, Internet of Service, e a Automação de Serviços Digitais (Buuse \& Kolk, 2019). Há etapas para as cidades tornarem-se inteligentes, como as estratégias inteligentes, os projetos inteligentes e o desempenho inteligente da estrutura e infraestrutura destas cidades (Camboim et al., 2019). Um aspecto oportuno é refletir quanto do Produto Interno Bruto (PIB) das cidades inteligentes que é alocado como recursos ou investimentos para projetos de modernização desse sistema de transporte urbano de massa, e como isso gera retorno às pessoas pelo serviço prestado, em oferta de tecnologias e diversificação do transporte urbano.

- Evidências pelo critério dos Desafios dos Sistemas de Transporte Urbano de Massa: Uma questão desafiadora sobre a análise da triangulação dos dados é a possível verificação se esses modos de transporte urbano de massa atendem a quem mais precisa, que seriam as regiões periféricas das áreas urbanas e metropolitanas, já que o conceito de cidades inteligentes traz em si o conceito de uma cidade ideal que consiga suprir as necessidades das pessoas através de indicadores, como a mobilidade inteligente. Esses transportes levam para onde? Todas as áreas centroides ou periféricas dessas cidades têm acesso a esse transporte? Esse transporte urbano prejudica a qualidade de vida das pessoas como um todo em áreas urbanas e 
metropolitanas? Existem restrições sobre o transporte público para os usuários? Ao longo da história, os sistemas de transportes de massa evoluíram, porém, se por um lado existe a busca pela utilidade no transporte por atributos (Garber \& Hoel, 2002; Hoel et al., 2008), por outro, existe também a busca pela escala de desutilidade do transporte (Raymundo \& Reis, 2018). Neste estudo pode-se refletir que os atributos do transporte urbano como, por exemplo, conforto, tempo, custo entre outros, podem ser alcançados em níveis satisfatórios com as tecnologias disponíveis e suas fontes de energia que podem ou não ter eficiência energética e serem mais ou menos poluidoras.

Os efeitos de um crescimento populacional desregrado nas cidades podem ser desastrosos para o ambiente e para os cidadãos, afetando sua qualidade de vida e a serventia e eficácia das operações nas cidades (Akande et al., 2019).

\section{Considerações Finais}

Este estudo de caso múltiplo apresenta que as cidades de Madri, Paris, São Paulo e Buenos Aires caminham para o uso de tecnologias da quarta revolução industrial ou da Indústria 4.0, associadas aos Intelligent Transportation Systems (ITS) na busca pela digitalização dos processos de mobilidade urbana, para redução dos impactos ao meio ambiente e melhoria da comunicação nos serviços de transporte de massa. Entretanto, a diferença entre as cidades europeias de Madri e Paris e as cidades sul-americanas de São Paulo e Buenos Aires está no volume de tecnologias inteligentes e sustentáveis disponíveis. Independentemente se é em maior ou menor grau de concentração no sistema de transporte e na sua composição, as tecnologias disponíveis afetam sensivelmente o indicador de mobilidade inteligente nas cidades. Analisando os indicadores socioeconômicos, econômicos, o ranking das cidades inteligentes e o estudo de caso múltiplo aplicado, o resultado alcançado por esta pesquisa revela que essa classificação tecnológica do transporte de massa para essas cidades inteligentes contribuem para conscientização do uso das tecnologias ao usuário, melhorando a mobilidade inteligente, e para os investimentos na infraestrutura do sistema de transporte de massa.

Considera-se que o estudo expôs para as cidades inteligentes estudadas a necessidade dos investimentos contínuos em diversificação dos modos de transporte de massa, aplicação concentrada de tecnologias em áreas urbanas e metropolitanas, utilização de maneira adaptativa e disruptiva em sua oferta para as pessoas/cidadãos que desempenham o papel de usuários desses serviços.

A limitação sobre o tema aponta a precariedade da variação de fontes de energia renováveis ofertadas que podem trazer como consequência uma sustentabilidade mais ou menos limpa ou poluente para o transporte público. Um ponto fundamental é que para classificar um sistema de transporte urbano de massa inteligente e sustentável é necessário traçar um paralelo entre as tecnologias que foram aplicadas, sejam elas da terceira revolução industrial, como os Intelligent Transportation Systems (ITS), ou pelas tecnologias da quarta revolução industrial, como a Indústria 4.0. Para pesquisas futuras é sugerido explorar ciclos históricos ou estágios do uso de modos transporte de massa e suas tecnologias pela humanidade.

\section{Agradecimentos}

"O presente trabalho foi realizado com apoio da Coordenação de Aperfeiçoamento de Pessoal de Nível Superior Brasil (CAPES) - Código de Financiamento 001".

\section{Referências}

Akande, A., Cabral, P., Gomes, P., \& Casteleyn, S. (2019). The Lisbon ranking for smart sustainable cities in Europe. Sustainable Cities and Society, 44, 75487.

Alavi, A. H., Jiao, P., Buttlar, W. G., \& Lajnef, N. (2018). Internet of Things-enabled smart cities: State-of-the-art and future trends. Measurement, 129, 589606. 
Aletà, N. B., Alonso, C. M., Ruiz, R. M. A. (2017). Smart Mobility and Smart Environment in the Spanish cities. Transportation Research Procedia, 24, 163170.

Buenos Aires Ciudad BA. (2020). Gobierno de la Ciudad de Buenos Aires. https://www.buenosaires.gob.ar/

Burch, I., \& Gilchrist, J. (2018). Survey of Global Activity to Phase Out Internal Combustion Engine Vehicles. https://heclimatecenter.org/wpcontent/uploads/2020/03/Survey-on-Global-Activities-to-Phase-Out-ICE-Vehicles-update-3.18.20-1.pdf

Buuse, D., \& Kolk, Ans. (2019). An exploration of smart city approaches by international ICT firms. Technological Forecasting \& Social Change, 142, 220234.

Camboim, G. F., Zawislak, P. A., \& Pufal, N. A. (2019). Driving elements to make cities smarter: Evidences from European projects. Technological Forecasting \& Social Change, 142, 154-167.

C40 CITIES. (2021). https://www.c40.org/about

Consórcio Regional de Transporte de Madrid. (2020). www.crtm.es

Country Economy. (2020). https://pt.countryeconomy.com/

Denzin, N., \& Lincoln, Y. (2000) Handbook of qualitative research. (2a ed). Thousand Oaks: Sage.

Dimitrakopoulos, G., Uden, L., \& Varlamis, I. (2020). Intelligent transport systems and smart mobility. Magazine The Future of Intelligent Transport Systems, 1st Edition. Elsevier, Amsterdã, 272.

Erkollari, A., Birgit, O. (2018). Sustainable cities need smart transportation: The Industry 4.0 transportation matrix. Sigma J Eng \& Nat Sci 9 (4), 359-370.

Flick, U. (1992). Triangulation revisited: strategy of validation or alternative? Journal for the Theory of Social Behavior. 22 (2), pp. $175-197$.

Garber, N. J., \& Hoel, L. A. (2002). Traffic and Highway Engineering. Third Edition. Brooks/Cole.

Hoel, L. A., Garber, J. N., \& Sadek, A. W. (2008). Transportation Infrastructure Engineering: A Multimodal Integration, SI Version, 1st Edition. Cengage Learning.

Holland, S. P., Mansur, E. T., Muller, N. Z., \& Yates, A. J. (2021). The environmental benefits of transportation electrification: Urban buses. Energy Policy. 148 .

IESE. (2020). IESE Cities in Motion INDEX 2020. Business School University Navarra. https://citiesinmotion.iese.edu/indicecim/?lang=en

International Energy Agency (IEA). (2020). Global EV Outlook 2020: Entering the decade of electric drive? Clean Energy Ministerial, and Electric Vehicles Initiative (EVI). https://www.iea.org/reports/global-ev-outlook-2020

Instituto Nacional de Estadística. (2020). Datos de la ciudad de Madrid. Https://Www.Ine.Es/

Instituto Nacional de Estadística y Censos Republica Argentina. (2020). Dados da Cidade de Buenos Aires. https://www.indec.gob.ar/

Kneib, E. C., \& Tedesco, G. M. I. (2010). Sustentabilidade energética do transporte público urbano: diretrizes para o Brasil. Revista Bibliográfica de Geografía y Ciencias Sociales, (8)881, 1-13.

Kumar, H., Singh, M. K. S., Gupta, M.P. G., \& Madan, J. (2019). Moving towards smart cities: Solutions that lead to the Smart City Transformation Framework. Technological Forecasting \& Social Change.

Lara, A. P., Moreira Da Costa, E., Furlani, T. Z., \& Yigitcanlar, T. (2016). Smartness that matters: towards a comprehensive and human-centred characterisation of smart cities. Journal of Open Innovation: Technology, Market, and Complexity, 2(1). doi:10.1186/s40852-016-0034-z.

Paris. (2020). https://www.paris.fr/

Plan Paris $360^{\circ}$. (2020). Https://Pt.Parismap360.Com/Mapa-Onibus-Paris\#Map42

Nowacki, G. (2012). Development and Standardization of Intelligent Transport Systems. International Journal on Marine and Safety of Sea Transportation, (6)3, 403-411.

Portal web del Ayuntamiento de Madrid. (2020).

Movilidad y Transportes (2020). https://www.madrid.es/portal/site/munimadrid

Prefeitura de São Paulo. (2020). www.capital.sp.gov.br

PricewaterhouseCoopers (2020). Global City GDP Ranking 2008-2025. https://pwc.blogs.com/files/global-city-gdp-rankings-2008-2025.pdf

Raymundo, H., \& Reis, J. G. M. (2018). Measures for Passenger-Transport Performance Evaluation in Urban Areas. Journal of Urban Planning and Development (3)144, 1-14.

Sacomano, J. B., Gonçalves, R. F, \& Bonilla, S. H. (2018). Indústria 4.0: Conceitos e Fundamentos. Blucher. 
Research, Society and Development, v. 10, n. 13, e37101320634, 2021

(CC BY 4.0) | ISSN 2525-3409 | DOI: http://dx.doi.org/10.33448/rsd-v10i13.20634

Tranfield, D., Denyer, D., \& Smart, P. (2003). Towards a Methodology for Developing Evidence-Informed Management Knowledge by Means of Systematic Review. British Journal of Management, 14(3), 207-222. doi:10.1111/1467-8551.00375.

Yin, R. K. (2014). Case Study Research Design and Methods (5th ed.). Sage, 282. 